\title{
A Taxonomy for Task Allocation Problems with Temporal and Ordering Constraints
}

\author{
Ernesto Nunes, Marie Manner, Hakim Mitiche, Maria Gini \\ Department of Computer Science and Engineering, University of Minnesota, 4-192 Keller \\ Hall, 200 Union St, Minneapolis, MN 55455
}

\begin{abstract}
Previous work on assigning tasks to robots has proposed extensive categorizations of allocation of tasks with and without constraints. The main contribution of this paper is a specific categorization of problems that have temporal and ordering constraints. We propose a novel taxonomy that emphasizes the differences between temporal and ordering constraints, and organizes the current literature according to the nature of those constraints. We summarize widely used models and methods from the task allocation literature and related areas, such as vehicle routing and scheduling problems, showing similarities and differences.
\end{abstract}

Keywords: task allocation, taxonomy, multi-robot coordination, temporal constraints, time-extended assignments

\section{Introduction}

What is multi-robot task allocation? Think of a shipping company that sells an item every hour; a robot at the warehouse could receive that order, fetch the item, pack it, and prepare it for pick-up by a postal service. What happens when the company sells 20 items every hour? What about 20 items every minute? What about 20 items a second? Amazon, a popular shopping website, sold 36.8 million items on an especially popular shopping day in 2013. With 426 items ordered per second that day, a single robot would be hard-pressed to keep up with the orders. If the warehouse used a large team of robots, each robot would have to plan an efficient route through the warehouse to fetch items for shipping without colliding with other robots, without taking items that another robot is handling, all while planning its route around fetching items that are out-of-stock but will be restocked soon.

Allocation of tasks with constraints on when, where, and in what order they need to be done is an important class of problems with many real-life

Email addresses: enunes@cs.umn.edu (Ernesto Nunes), manner@cs.umn.edu (Marie Manner), h.mitiche@gmail.com (Hakim Mitiche), gini@umn.edu (Maria Gini)

Preprint submitted to Robotics and Autonomous Systems

October 3, 2016

C) 2016. This manuscript version is made available under the Elsevier user license http://www.elsevier.com/open-access/userlicense/1.0/ 
applications, such as warehouse automation, pickup and delivery, surveillance at regular intervals, space exploration, and search and rescue.

Gerkey and Mataric [2004] have proposed a widely accepted taxonomy for multi-robot task allocation (MRTA) problems, which is based on the main characteristics of robots, tasks, and time, as follows:

- Single-task robots $(S T)$ vs. multi-task robots $(M T)$ : ST robots can do at most one task at a time, while MT robots can work on multiple tasks simultaneously.

- Single-robot tasks (SR) vs. multi-robot tasks (MR): SR tasks require exactly one robot in order to be completed, while multiple robots are needed to complete an MR task.

- Instantaneous (IA) vs. time-extended (TA) assignments: In IA, tasks are allocated as they arrive, while in TA, tasks are scheduled over a planning horizon.

The iTax taxonomy [Korsah et al., 2013] adds a level above Gerkey's taxonomy, focusing on interrelated utilities and constraints among tasks, both for individual robots and across robots, and for complex tasks which can be partitioned in many different ways into simpler tasks. We are interested in considering a different aspect, specifically the temporal and ordering constraints that might exist between tasks.

The nature of the temporal constraints in MRTA problems is very broad; for example, in search and rescue domains tasks are discovered over time and have to be done as quickly as possible. In dynamic environments, robots might arrive late to some tasks and might miss some. On the other hand, some surveillance tasks require not to arrive late to tasks. In some cases tasks need to be executed in a specific order, such as in urban disaster scenarios in which police must clear blockades from roads before ambulances can travel to carry injured people. Some tasks may need to be done concurrently, as in surveillance where robots have to track people while avoiding obstacles.

Our main contribution is an extension to Gerkey and Matarić [2004] taxonomy, where we expand the time-extended (TA) part to include temporal and ordering constraints. We consider temporal constraints expressed in the form of time windows (TA:TW) and ordering constraints expressed in the form of synchronization and precedence constraints (TA:SP). To emphasize the importance of this class of problems we propose the definition of multi-robot task allocation problems with temporal and ordering constraints as MRTA/TOC.

In this paper we also explore the following research questions:

- What models and methods from related research areas can be applied to this class of problems?

- What are the main types of temporal and ordering constraints used in multi-robot task allocation? 
- What are the most commonly used optimization objectives? Are they predominantly temporal-based, distance-based, or multi-objective?

- What are the main solution approaches used for the different parts of the taxonomy? are the methods deterministic or stochastic? are the temporal constraints hard or soft? are the algorithms centralized or distributed?

- Which questions for this class of problems have been answered well, and which remain largely open?

We begin by summarizing the terminology used in the paper, and defining the class of multi-robot task allocation problems with temporal and ordering constraints (MRTA/TOC) in Section 2. In Section 3 we relate this class of problems to problems in other areas, setting the ground for our exploration of models and methods in those areas. In Section 4 we present temporal and ordering models. In Section 5 we review the most common optimization objectives considered in the literature. Our taxonomy is introduced in Section 6 . Task execution and the dynamics therein are discussed in Section 7. Solution approaches are outlined in Section 8. We discuss open issues, future directions, and final thoughts in Section 9.

\section{MRTA/TOC: Multi-robot Task Allocation with Temporal and Or- dering Constraints}

\subsection{Terminology and Abbreviations}

We define the terminology we use informally as follows:

- A robot is an autonomous agent responsible for performing some actions. Alternative names for robots are physical agents, unmanned vehicles, and rovers. Robots in MRTA are typically modeled as holonomic or point robots, since the focus is not on low level control of robot motion.

- A team is a set of robots that work together. A team is often called a coalition when it is dynamic, i.e. formed to do some tasks and disbanded after that [Parker and Tang, 2006].

- A task is an action to be performed, also referred to as a work unit, activity, waypoint, or customer request. In some scheduling literature tasks are divided into jobs [Davis and Burns, 2011], but in other cases jobs are made of tasks [Balas et al., 2008].

- A time window is a time interval, which starts with the earliest time a task can start, and ends with the latest time the task can end. If the earliest time is not given, the latest time is referred to as the deadline. A time window is said to be closed if both start and end times are given.

- Synchronization constraints specify temporal constraints among tasks, for instance, they have to start at the same time. 
- Precedence constraints specify partial ordering relationships between pairs of tasks, for instance, a task has to be completed before another task can start.

- A schedule is a timetable in which each task has a specific time to start, end, or both. In some cases each robot has its own individual schedule (e.g., [Nunes and Gini, 2015]), while in others all robots share a single schedule.

- The makespan is the time difference between the end of the last task and the start of the first task.

- A route is a sequence of locations to visit. Routes and schedules are often used interchangeably, but schedules always concern time, while routes concern physical locations.

- A task release refers to a task becoming available for execution. Task release can be deterministic if the release time is known upfront, dynamic if the release time is stochastic, or sporadic if it is governed by unknown probabilities; task release is periodic when the same task is released at regular intervals.

We use the following acronyms:

- MRTA/TOC for Multi-Robot Task Allocation with Temporal and Ordering Constraints.

- MIP for Mixed Integer Programming, and MILP if the objective function and constraints are linear.

- TOPTW for Team Orienteering Problem (TOP) with Time Windows.

- VRPTW for Vehicle Routing Problem (VRP) with Time Windows.

- JSP for job-shop scheduling problems.

\subsection{Problem Formulation}

We assume there is a finite set of robots and of tasks. A robot may have a location, speed, route, and/or schedule. A task may have a location, earliest start, latest finish time, expected duration, cost, demand, and reward.

Constraints on tasks can be in the form of time windows, which specify the window of time when the task can be done. It is assumed that a robot which reaches a task location before the earliest start time will wait until the earliest start. Ordering constraints specify a dependency between pairs of tasks. They are usually represented as directed acyclic graphs, where each node in the graph represents a task, and each edge indicates a precedence constraint. In addition to precedence constraints, ordering constraints can be synchronization constraints, which specify, for instance, that two tasks have to start at the same 
time or that a task has to start a specific amount of time after another task finishes.

We use the term synchronization constraints when there is a specific time involved and precedence constraints when it is only an ordering constraint. In the Operation Research literature, the term General Precedence Relationship (GPR) is used to indicate both those types of constraints, i.e., task $j$ has to start within a time window after the completion of task $i$, or task $j$ can start any time after the end of task $i$ [Monma, 1981, Brucker et al., 1999].

The objective is to optimize some function of the cost (or reward) for doing the tasks for all the robots. Cost can be a temporal measure (e.g. makespan), or a spatial measure (e.g. distance traveled). Commonly used optimization objectives are described later in Section 5 .

\section{Connections with Other Problems}

Multi-robot task allocation (MRTA) started in earnest in the 90's, when researchers started pulling together teams of robots to accomplish multiple tasks. MRTA draws from a variety of areas in mathematics and operations research as well as computer science and robotics, including assignment problems, distributed computing, distributed AI, and scheduling.

The search for robust approaches to MRTA focused on how the robots perform in complex environments, leading researchers to add features like time windows for tasks, spatial constraints, and probabilistic and stochastic models to handle uncertainty. Solutions take different approaches, such as auctions, market-based planning, Markov Decision Processes, decentralized scheduling algorithms, and distributed constraint optimization.

In this paper we cover a subset of MRTA problems, which we call MRTA/TOC, to highlight the importance of temporal and ordering constraints among tasks and to shed light on how the inclusion of those constraints increases the complexity of task allocation.

Similar types of problems include the vehicle routing problem [Dantzig and Ramser, 1959], the job shop scheduling problem [Manne, 1960], and the team orienteering problem [Chao et al., 1996]. Overall, multi-robot task allocation diverges from each of these problems on key points, including assumptions on the number of robots, robot and task homogeneity, environment dynamics caused by failures or interference with other robots, and communication restrictions.

We are now prepared to discuss the relationship between MRTA/TOC problems and the vehicle routing problem with time windows (VRPTW), the team orienteering problem with time windows (TOPTW), and the job-shop scheduling problem (JSP).

\subsection{MRTA/TOC vs. VRPTW}

The vehicle routing problem with time windows (VRPTW) [Kolen et al., 1987, Desrochers et al., 1988, Solomon and Desrosiers, 1988, Toth and Vigo,

2002] studies problems which require solving allocation, routing, and scheduling 
subproblems simultaneously. Vehicles and robots are typically treated as points in space, ignoring kinematic constraints, but kinematic [e.g. Cheng et al., 2008, for unmanned aerial vehicles] and sometimes dynamic [Pecora and Cirillo, 2012, for ground vehicles] constraints can be considered.

The solutions to several variants of VRPTW, such as multi-depot [Polacek et al., 2004, Kang et al., 2005], dynamic and stochastic [Laporte et al., 1992, Pavone et al., 2011, Taş et al., 2013], and precedence and synchronization constrained [Bredström and Rönnqvist, 2008, Korsah et al., 2012], have often been extended to MRTA/TOC settings.

An example of VRP similarities is the online pickup and delivery problem with transfers, where a team of vehicles has to pick up a set of items at a location and deliver them to another location [Coltin and Veloso, 2014b]. This problem is a generalization of the pickup and delivery problem [Savelsbergh and Sol, 1995] which is well studied in operations research. However, the proposed solution is a typical MRTA approach. The authors combine a centralized temporal planner, which creates the initial schedules, with auctions, which are used to repair the plans when delays or failures occur. In the same vein, Korsah et al. [2012] studied a MRTA problem that can be framed as a vehicle routing problem with temporal, precedence and synchronization constraints. They proposed a MILP model and an optimal Branch-and-Price solution.

Despite their similarities, these problems differ in some ways. First, VRPTW assumes an infinite number of vehicles is always available, with a few exceptions [e.g. Lau et al., 2003]). This assumption is not practical in robotic systems where the number of robots is usually fixed and can even decrease due to failures. VRPTW problems usually assume that all vehicles start from the same depot and return to the depot after work. In MRTA/TOC problems, robots may start at different locations and do not need to return to their initial locations. VRPTW problems mostly assume homogeneous vehicles with respect to their capabilities and capacities [for exceptions, see Dondo and Cerdá, 2007, Bettinelli et al., 2011], while in MRTA/TOC robots are not necessarily homogeneous and their capacities and types can differ [Schneider et al., 2005, Xu et al., 2005, Ponda, 2012]. Lastly, unlike VRPTW problems, in MRTA/TOC problems communication is important and often constrained. In [Mercker et al., 2010] the communication graph is unknown (hence the algorithm does not always converge), while in [Ponda, 2012] the communication graph is maintained by specialized robots or robots not working on a task that act as communication relays. Jackson et al. [2013] and Smith and Bullo [2007] proposed distributed algorithms that converge using only local communication.

\subsection{MRTA/TOC vs. TOPTW}

In the team orienteering problem with time windows (TOPTW), an origin and destination pair is given, and the goal is to search for control points to visit between the origin and destination such that the profit (or score function) is maximized while respecting all the constraints. Each control point is associated with a profit (or score), and each edge connecting control points is weighted by the cost of moving between the control points [Labadie et al., 2012]. Control 
points are equivalent to tasks for robots in MRTA. Profit is often computed as the difference between the task's reward and the cost a robot incurs in reaching and performing the task.

When TOPTW uses the same point for origin and destination we have subtours similar to those for VRPTW problems. One application of TOPTW problems, dial-a-ride, has gained popularity in MRTA/TOC [e.g. Rubinstein et al., 2012, Coltin and Veloso, 2014a], In dial-a-ride, the problems are over-constrained [Carrabs F., 2007, Cordeau and Laporte, 2007], which means that not all the tasks can be performed, and thus the goal is to find the subset of tasks that maximizes the total profit [Rubinstein et al., 2012].

\subsection{MRTA/TOC vs. JSP}

The job-shop scheduling problem (JSP) is concerned with allocating groups of activities, called jobs, to a set of machines with the goal of minimizing the cost of completing the jobs, alone or in combination with other objectives [Graham et al., 1979, Allahverdi et al., 2008]. The problem can be decomposed into sequencing the activities and assigning start and end times to them (scheduling), which are solved simultaneously. Deterministic MRTA/TOC problems can be modeled as job-shop scheduling problems with setup times, deadlines, and precedence constraints [Cesta et al., 2000, Balas et al., 2008, Oddi et al., 2011]. In order to model MRTA/TOC problems as job-shop scheduling problems, tasks are treated as jobs and robots as machines. Simple tasks can be mapped to a job with only one activity, and complex tasks with subtasks to a job with multiple activities.

However, mathematical models for JSP do not apply directly to MRTA/TOC problems, because JSP does not account for travel time. Even when setup times are used in JSP, the setup time typically depends on the machine and not on the time needed for the job to reach the machine. The equivalent of travel time would be to use setup times that depend on the specific job [Korsah et al., 2013].

Modeling MRTA/TOC problems as JSP problems is most useful when models and methods developed for scheduling [Cesta and Oddi, 1996, Cesta et al., 1999, Lee et al., 2009, Shah et al., 2009] are combined with MRTA solution techniques. In [Gombolay et al., 2013] a centralized approach is proposed in which a central temporal network is used and integrated with a MILP-based planner yielding near optimal schedules. In the decentralized approach of Barbulescu et al. [2010] each robot forms its own simple temporal network [Dechter et al., 1991], encoding both temporal and precedence constraints in the network. To enforce precedence constraints a robot has to know which other robots depend on its schedule, so a high communication overhead is required to keep all robots up-to-date. The distributed approach in [Nunes and Gini, 2015] cuts down on communication costs by having each robot keep its own independent local temporal network and uses sequential single-item auction for allocation.

Having outlined the differences and similarities between MRTA/TOC and related problems, we now turn our attention to temporal and ordering constraints on MRTA problems. 


\section{Temporal Models}

Temporal models are critical in our taxonomy. We outline time interval models [Allen, 1983] in Subsection 4.1 and Simple Temporal Networks [Dechter et al., 1991] in Subsection 4.2. Time windows are presented in Subsection 4.3, precedence and synchronization constraints in Subsection 4.4, while in Subsection 4.5 we discuss the nature of temporal constraints.

\subsection{Relationships between time intervals}

In general terms, time can be modeled using points or intervals [Allen, 1983]. An example of a time point is $10 \mathrm{am}$, while an interval is a continuous set of values bounded below and above by some time point, for example [10 am-12 $\mathrm{pm}]$. When representing temporal constraints we may use either representation; however, the interval representation is much more common and is referred to as a time window.

The seminal paper by Allen [1983] proposed a set of relationships that hold between any two time intervals, as depicted in Fig. 1.

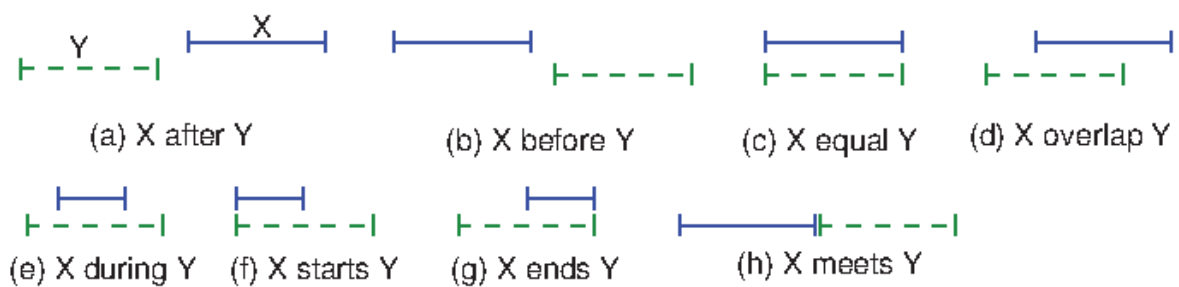

Figure 1: All possible relationships between pairs of time intervals [Allen, 1983]

While the relationships originally were described between qualitative time intervals, they are also useful to describe the ordering between quantitative time intervals. The relationships can be used to model partial or complete ordering constraints between tasks, - for example, task $X$ should be done before, after, or at the same time as task $Y$. The " $X$ before $Y$ " operator can be used to describe precedence constraints between tasks, while the " $X$ equal $Y$ " operator describes a synchronization constraint between the start and end points of two tasks.

\subsection{Simple Temporal Networks (STN)}

Equally influential is Dechter et al. [1991] approach, which proposed to represent a class of temporal constraints with a graph, called a simple temporal network (STN). An example is in Fig. 2.

Nodes represent time point variables or time events, and weighted edges represent inequality constraints between time points. To reduce computational complexity, this model requires exactly one constraint between pairs of time point variables. A solution to the scheduling problem can be computed in polynomial time using the Floyd-Warshall algorithm. In an STN the relationship 


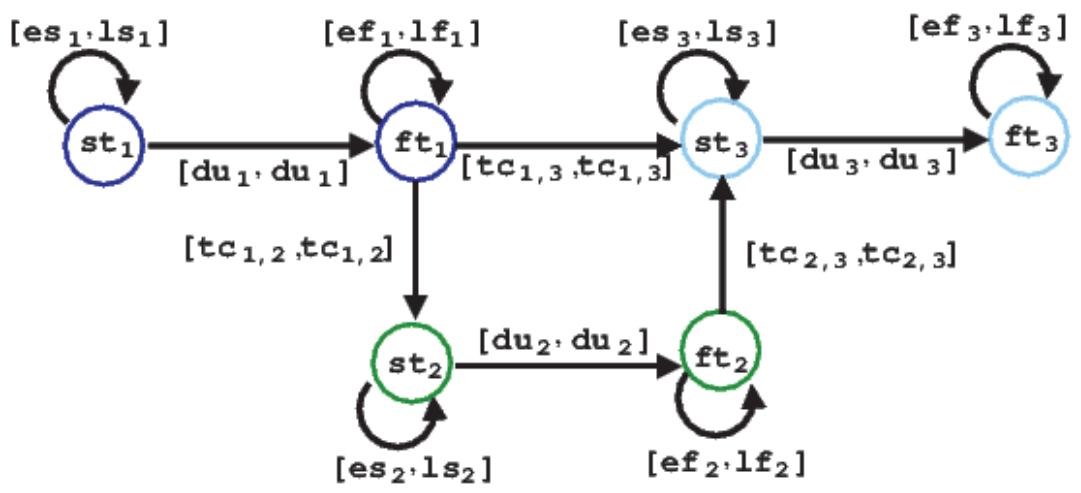

Figure 2: A simple temporal network with three tasks 1, 2 and 3, each with a time window. The self-loops on tasks indicate the absolute start and end times for the task. Task 1 is done first, and then there is a choice of doing 2 and 3 or just 3. st and $f t$ are the actual start and finish times for each task, es and $l s$ are the earliest and latest times tasks can start, similarly ef and $l f$ are the earliest and latest times tasks can finish, and $d u$ represent tasks' durations. $t c_{k, k^{\prime}}$ is a time cost defined as the sum of the travel and wait times, which constrains when the next task $k^{\prime}$ can be started.

between time windows can be represented by establishing constraints between start and finish times of tasks. While there are more complex models, for instance, disjunctive temporal constraints [Stergiou and Koubarakis, 2000] and Temporal Plan Networks [Block et al., 2006], in general they can be approximated by solving several simple temporal problems [Boerkoel and Durfee, 2013].

STNs are commonly used in MRTA/TOC problems [Barbulescu et al., 2010, Gombolay et al., 2013, Coltin and Veloso, 2014a, Nunes and Gini, 2015] because constraint consistency can be efficiently verified in polynomial time [Dechter et al., 1991, Xu and Choueiry, 2003, Planken et al., 2008]. An important feature of STNs is that new time points and constraints can be dynamically added in polynomial time [Cesta and Oddi, 1996, Coles et al., 2009], which is beneficial in dynamic domains where new tasks can appear and disappear.

STNs have been successfully extended to multi-agent settings [Hunsberger and Grosz, 2000, Boerkoel and Durfee, 2012, Boerkoel and Planken, 2012] and to scenarios with uncertainties. Vidal [1999] uses set bounded uncertainty to model duration uncertainty of temporal events in an STN, and introduces the STN with uncertainty (STNU). Tsamardinos [2002] and Fang et al. [2014] extend STNUs by modeling uncertainty as probabilities. The former attempts to minimize the risk of temporal inconsistencies occurring, and the latter attempts to bound the probability of not meeting a schedule.

\subsection{Time Window Constraints}

A time window is a temporal interval constraint on the start and finish time of a task. They are commonly used for scheduling problems. A time window has a lower bound value, usually the task's earliest start time, and an upper 
bound value, usually the task's latest finish time. A task can also have a latest start time and an earliest finish time, resulting in a time window of the form [earliest start time, latest start time, earliest finish time, latest finish time]. This representation implicitly provides an upper bound to the task duration. When the earliest and latest start times are the same, the time window specifies only a start time. Same for finish time. If only a start time is given the finish time is assumed to be the end of the scheduling horizon; similarly if only a finish time is given the start time is assumed to be the beginning of the scheduling horizon.

Time window constraints can be used to model many types of temporal relationships among tasks. For instance, deadline constraints [Ramchurn et al., 2010b, Amador et al., 2014, Luo et al., 2015] only impose constraints on the latest time robots can arrive to a task before the task expires. In some cases, time windows are used only to specify the interval in which a task has to start [Ponda et al., 2010].

The use of time windows for auction-based task allocation to agents was pioneered in the MAGNET system, which proposed various task allocation algorithms [Collins et al., 2000, Collins and Gini, 2006].

The flexibility in the temporal constraint representation, together with the temporal relationships between time windows (see Section 4.1) makes time windows a powerful modeling tool for temporal constraints. However, this flexibility increases the search space because there are multiple ways of scheduling a task within its time window.

Task allocation problems with time windows are generally, except for a few special cases (e.g. [Melvin et al., 2007]), NP-hard [Solomon, 1986], and finding a feasible solution is NP-complete [Savelsbergh, 1985]. Efficient approximation algorithms are hard to deisgn. This is different from task allocation problems without constraints, which have a constant factor approximation for positive valued submodular utility functions [Segui-Gasco et al., 2015].

\subsection{Precedence and Synchronization Constraints}

Precedence constraints specify a partial or total order for the tasks, without providing a specific time window for each task. Time windows can be used to specify implicitly precedence or synchronization constraints, but in general they are not sufficient. Two time windows with the same start time do not necessarily indicate a synchronization constraint. Time windows that overlap are not sufficient to specify precedence constraints.

Solutions to task allocation problems with precedence or synchronization constraints might contain assignments to different robots of tasks that depend on each other. This creates cross-schedule dependencies among robots [Jones et al., 2011, Korsah, 2011]. These dependencies are undesirable because exogenous events during execution affecting one robot will also affect the robots that depend on it.

Precedence and synchronization constraints impose partial ordering between tasks, which can be used to eliminate candidate solutions that violate the ordering. Instead, tasks with time windows are independent of each other and 


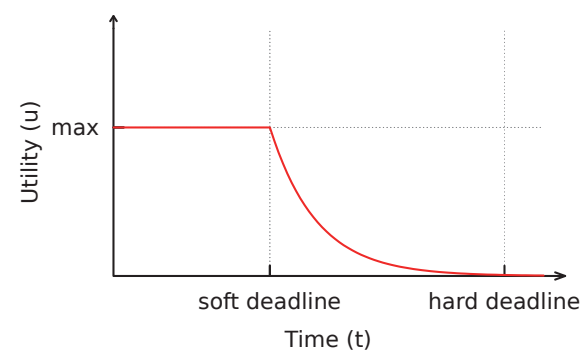

(a) soft deadline utility function

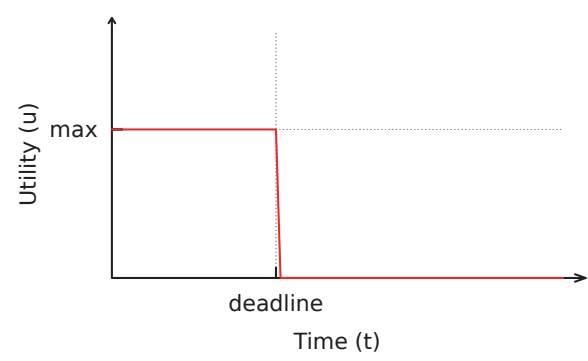

(b) hard deadline utility function

Figure 3: Utility of soft deadlines vs. hard deadlines. The maximum utility is earned before the deadline. An exponentially decaying utility can be gained if the task finishes between the soft and hard deadlines (case (a)). No utility is gained after the (hard) deadline (case (b)).

can be performed in any order, as long as the robots can reach the tasks and execute them within the tasks' time windows.

\subsection{Hard vs. Soft Temporal Constraints}

Temporal constraints can be characterized as hard or soft. Hard temporal constraints cannot be violated [Borning et al., 1992]. They are used in MRTA/TOC and related areas for tasks like surveillance, routing for perishable goodies, and order fulfillment by warehouse robots. Soft temporal constraints allow some temporal constraints to be violated or some tasks to be skipped entirely, but typically the robot incurs a penalty for doing so [Domshlak et al., 2006]. The penalty incurred may differ depending on which constraint was violated; for example, finishing tasks late may be penalized more severely than doing tasks early Ponda et al. [2010].

Fig. 3 illustrates the difference between a soft deadline utility function (left) and a hard deadline utility function (right).

In some problems (e.g. Robocup Search and Rescue [Kitano and Satoshi, 2001]) tasks expire at an unknown time (e.g. [Scerri et al., 2005, Ramchurn et al., 2010b]). In this case the utility function (left plot in Fig. 3) drops to zero at the unknown deadline. Agents have to work as fast as possible to complete tasks before they expire. If people are found quickly they can be rescued before they die. If enough firefighters work on a fire, the fire can be extinguished before the entire city burns [Parker and Gini, 2014].

Common types of soft temporal constraints include:

1. tasks can be started early and/or finish late with some penalty (called soft constraints in real time system terminology);

2. deadlines need to be satisfied only with some probability [Zheng and Woodside, 2003];

3. a number of consecutive tasks or some percentage of the tasks can be skipped [Bernat et al., 2001] without penalty (called weakly hard constraints in the real time systems terminology); 
4. some tasks can be done late without reward, or skipped without penalty (called firm tasks in [Bernat et al., 2001]);

5. positive and negative preferences can be used as soft constraints [Bistarelli et al., 2007, Hoogendoorn and Gini, 2009].

Relaxing some of the temporal constraints and penalizing infeasible solutions so obtained has been shown to be effective in speeding up the computation and improving the quality of heuristic search. Vidal et al. [2014] have produced in this way some best known solutions for benchmark problems in vehicle routing.

The weakly hard constraints from real time systems are not widely used in MRTA, even though they transfer quite sensibly into MRTA problems. Four types of weakly hard constraints have been considered in the literature: (1) making any $n$ in $m$ deadlines, (2) making $n$ sequential deadlines in $m$, (3) missing any $n$ in $m$ deadlines, and (4) missing $n$ sequential deadlines in $m$. In this way, any regularly scheduled sequence of tasks can allow some missed deadlines without penalty, while still allowing the agent responsible for those tasks to schedule and make most of its deadlines. Agricultural drones, for example, may have regularly scheduled sampling, such as fertilization, weed picking, or soil testing responsibilities that allow to skip a few deadlines.

Real world robot-task assignment problems might demand periodic tasks. A Mars rover, for example, has a regularly scheduled self-maintenance period, as well as periodic deadlines to finish uploading data or downloading instructions. These deadlines are usually hard deadlines, so the robot can shut down overnight and clear memory caches; other regularly scheduled robotic activities are not so sensitive to the time of execution.

In weakly hard systems with periodic task release, the distribution of met and missed deadlines in a time period is precisely bounded [Bernat et al., 2001]. Other approaches to skipping some deadlines for periodic tasks include degradation policies in overloaded systems [Beccari et al., 1999] or exploiting skips to improve response time for aperiodic tasks [Caccamo and Buttazzo, 1997].

Having discussed temporal models and constraints, and the nature of ordering constraints, we switch focus to optimization objectives. Determining these objectives is another important aspect to consider when building models for MRTA/TOC problems.

\section{Optimization Objectives}

Applications of MRTA/TOC problems require the robots to achieve a given optimization objective. In the rest of this paper we will refer to $f(\cdot)$ as a generic function representing one of these objectives. There can be a single or multiple objectives [Jozefowiez et al., 2008]. Depending on the deterministic or stochastic nature of the problem, objectives will either be over actual or expected values. Optimization objectives might require a quantity to be minimized, usually a cost [Chopra and Egerstedt, 2012, Gombolay et al., 2013, Nunes and Gini, 2015] or regret [Heap and Pagnucco, 2014, Wu and Jennings, 2014], or to be maximized, 
usually a score [Mercker et al., 2010, Ponda, 2012] or a reward [Koes et al., 2005, Melvin et al., 2007, Korsah et al., 2012]. Single optimization objectives may be of spatial nature (e.g. minimize total distance traveled) or of temporal nature (e.g. minimize makespan).

Common optimization objectives for MRTA/TOC problems include:

- MiniSUM, i.e. minimize the sum of the robot path costs over all the robots [Lagoudakis et al., 2005]. Minimizing the distance traveled is common [e.g. Coltin and Veloso, 2014b, Chopra and Egerstedt, 2012, MacKenzie, 2003]) but some instead minimize a time measure over robot paths [e.g. Barbulescu et al., 2010, Heap and Pagnucco, 2014]).

- MiniMAX, i.e. minimize the maximum path cost of a robot over all the robots [Lagoudakis et al., 2005]. Instead of minimizing the maximum path cost, a similar objective function is to minimize the makespan, i.e. the time difference between the start of the first and the end of the last task [Graham et al., 1979].

- MiniAVE: i.e. minimize the average per task cost of the path over all the tasks. The per task cost is the cost of the path from the initial location of the robot to the task location [Lagoudakis et al., 2005]. This is known as the Traveling Repairman Problem [Fakcharoenphol et al., 2007], where the objective is to minimize the wait time of the customers (or tasks) for a repairman (or robot).

- Minimize lateness or tardiness, which is the difference between the earliest start time of a task and the actual arrival time of the robot [Beck and Refalo, 2003, Ponda, 2012, Rubinstein et al., 2012]. A similar objective is to minimize the idle time of the robots [Hasgül et al., 2009].

- Maximize the number of tasks completed [Lau et al., 2003, Colorni and Righini, 2001] or minimize the number of tasks missed [Hasgül et al., 2009].

- Minimize the number of robots used. This is common in vehicle routing problems, where the number of vehicles available is unlimited [Luo and Schonfeld, 2007, Bräysy and Gendreau, 2005a, Desrochers et al., 1988].

- Maximize profit, measured as the difference between the reward of tasks and their respective costs [Melvin et al., 2007, Korsah et al., 2012], or as the team utility [Koes et al., 2005, Ponda, 2012, Amador et al., 2014].

While not extensively covered here, multi-objective problems are common [Jozefowiez et al., 2008], especially when objectives are combined through linear aggregation. For example, makespan and distance are minimized in [Ponda, 2012, Nunes and Gini, 2015], while workspace overlap is also minimized in [Gombolay et al., 2013]. In [Alighanbari et al., 2003] a multi-objective function minimizes the maximum and average task completion times, as well as total idle times. 


\section{Taxonomy}

We are now ready to introduce our extensions to the taxonomy of Gerkey and Matarić [2004] focusing on time-extended assignments, in which robots build schedules for the tasks. We categorize the literature according to time window and precedence constraints. We add the following new axes to the taxonomy:

- Time Window $(T W)$ vs. Synchronization and Precedence (SP) constraints. Within each subcategory, when appropriate, we further distinguish:

(a) hard temporal constraints vs. soft temporal constraints. Hard temporal constraints require that no temporal constraint is violated, while soft temporal constraints allow some violations with a penalty.

(b) deterministic vs. stochastic models. In deterministic models the output of the model is completely determined by the initial conditions, while stochastic models assume a model of the uncertainty is available. Despite the importance of uncertainty in robotics, most MRTA models are deterministic and deal with uncertainty only at execution time.

We now illustrate our taxonomy in terms of single- vs. multi-task robots (SR vs. MR), single- vs. multi-robot tasks (ST vs. MT), and time windows vs. synchronization and precedence constraints (TW vs. SP). We begin with the least complex problem settings, in which single-task robots get allocated single-robot tasks.

\subsection{ST-SR-TA:TW - Single-Task robots, Single-Robot tasks, Time-extended Assignments: Time Windows}

\subsubsection{Hard Temporal Constraints}

Deterministic allocations. Deterministic ST-SR-TA:TW problems typically assume that there are more tasks than robots and the tasks are known in advance. They require time-extended assignments and have time window constraints on the tasks.

These problems are composed of three intertwined subproblems: (1) an assignment subproblem, to find the assignment of tasks to robots that optimizes the given objective function $f(\cdot) ;(2)$ a task sequencing subproblem, to find feasible orderings of tasks that result in optimal assignments, and (3) a scheduling subproblem, to assign times to tasks in a way that optimizes $f(\cdot)$.

Tasks have to be scheduled so that no constraint is violated. Temporal constraints are violated when robots do tasks at times that are not consistent with the temporal constraints of the tasks. Assignment violations occur when two or more robots are assigned to the same task, or two or more tasks are scheduled to be done at the same time by the same robot.

We summarize the notation we use in Table 1.

A mixed integer linear programming formulation is shown in Fig. 4. Since each robot starts at its initial location, we create a dummy task for each robot 


\begin{tabular}{ll}
\hline & \multicolumn{1}{c}{ Robots } \\
\hline$A$ & set of robots \\
$Q_{a}$ & $\begin{array}{l}\text { robot in set } A \\
\text { capacity, or maximum workload, of robot } a\end{array}$ \\
\hline$K$ & \multicolumn{1}{c}{ Tasks } \\
$k$ & set of tasks \\
$E S_{k}$ & task in set $K$ \\
$L S_{k}$ & latest start time of task $k$ \\
$E F_{k}$ & earliest finish time of task $k$ \\
$L F_{k}$ & latest finish time of task $k$ \\
$S_{k}$ & actual start time of task $k$ \\
$F_{k}$ & actual finish time of task $k$ \\
$D U R_{k}$ & duration of task $k$ \\
$T T_{k, k^{\prime}}$ & travel time between tasks $k$ and $k^{\prime}$ \\
$W_{k}^{a}$ & workload for task $k$ when performed by robot $a$ \\
\hline \multicolumn{2}{c}{ Optimization } \\
\hline$f(\cdot)$ & generic optimization function \\
$x_{k}^{a}$ & indicator of assignment of task $k$ to robot $a$ \\
$o_{k k^{\prime}}^{a}$ & indicator that robot $a$ performs task $k^{\prime}$ directly after $k$ \\
$z_{k}^{a}$ & indicator that robot $a$ performs task $k$ last
\end{tabular}

Table 1: Notation used in the paper for tasks with time window constraints.

$a$ at its initial location. The dummy tasks encode the start positions of the robots. They all finish at time 0 . We indicate the set of all the tasks plus the dummy task at the start location of robot $a$ as $K_{a}^{+}=K \cup\{$ start location of $a\}$.

The last constraint $(\mathrm{j})$ means that this problem is not a mixed-integer program, but it could be replaced with $S_{k}-F_{k^{\prime}}-T T\left(k, k^{\prime}\right) \geq M\left(o_{k, k^{\prime}}^{a}-1\right)$ for a sufficiently large constant $M$. Any precedence constraints between tasks would need to be added to the constraints listed in Fig. 4,

The objective function $f(\cdot)$ in the optimization formulation can be a cost function to be minimized [e.g. Gombolay et al., 2013]), or a value function to be maximized [e.g. Koes et al., 2005]). It can also be single or multi-objective.

For instance, to minimize the makespan the optimization objective would be:

$$
\operatorname{minimize}_{x_{k}^{a}, o_{k k^{\prime}}^{a}, z_{k}^{a}, S_{k}, F_{k}} \max _{a \in A} \max _{k \in K_{a}^{+}} F_{k}
$$

Advances in MILP formulations for VRPTW [Barnhart et al., 1998, Feillet, 2010] and more recently for MRTA problems [Korsah et al., 2012] have proposed formulations based on set covering and set partitioning. These formulations assign routes, instead of tasks, to robots. The allocation problem is decomposed into what is known as the master problem, and a pricing subproblem. One of the advantages of such formulations is that the master problem can be restricted 
minimize or maximize $f($.

subject to
(a) $\forall a \in A$, and $\quad x_{k}^{a}=1$
$k=$ start of $a$
(b) $\forall k \in K$
(c) $\forall a \in A$
(d) $\forall a \in A, k^{\prime} \in K$
$\sum_{a \in A} x_{a}^{k}=1$
$\sum_{k \in K} W_{k}^{a} x_{a}^{k} \leq Q_{a}$
$\sum_{k \in K_{a}^{+}}^{a} o_{k, k^{\prime}}^{a}=x_{k^{\prime}}^{a}$
(e) $\forall a \in A, k \in K_{a}^{+}$
$\sum_{k^{\prime} \in K} o_{k, k^{\prime}}^{a}+z_{k^{\prime}}^{a}=x_{k}^{a}$
Every $a$ has an initial
... dummy task.
(f) $\forall a \in A$
$\sum_{k \in K_{k}^{a} z_{k}^{a}}=1$
(g) $\forall k \in K$
$E S_{k} \leq S_{k} \leq L S_{k}$
(h) $\forall k \in K$
$E F_{k} \leq F_{k} \leq L F_{k}$
Every task gets a robot.
No robot exceeds its capacity.
Every task has exactly
... one predecessor.
(i) $\forall k \in K$
$F_{k}-S_{k} \geq D U R_{k}$
Every task except a last task
... has exactly one successor.
(j) $\forall a \in A, k \in K_{a}^{+}$, and $k^{\prime} \in K$
(k) $\forall a \in A, k \in K \quad x_{k}^{a} \in\{0,1\}$
(l) $\forall a \in A, k \in K_{a}^{+}, \quad o_{k k^{\prime}}^{a} \in\{0,1\}$ and $k^{\prime} \in K_{a}$
(m) $\forall a \in A, k \in K_{a}^{+} \quad z_{a}^{k} \in\{0,1\}$ Every robot has a last task.
Start time in valid range.
Finish time in valid range.
Task time is long enough.
$o_{k . k^{\prime}}^{a}\left[S_{k}^{\prime}-F_{k}-T T\left(k, k^{\prime}\right)\right] \geq 0$ Time between 2 consecutive
... tasks allows for travel time.
Indicator: $a$ does task $k$
Indicator: $a$ does task $k^{\prime}$
...right after $k$.
Indicator: $k$ is $a$ 's last task.

Figure 4: Mixed integer linear programming formulation of allocation of tasks with time windows. $f($.$) is the objective function, which could represent cost, or makespan, or reward.$

to evaluating subsets of tasks at a time, instead of the entire set of tasks. Pricing subproblems solve temporally constrained shortest path problems rooted at robot locations, in which routes can be computed via heuristic methods, such as D* lite as in [Korsah et al., 2012]. Such formulations benefit from the insight that for very large problems many routes are not part of any optimal solution. Thus, selectively incrementing candidate routes decreases computational and memory costs. Feillet [2010] provides a technically rigorous overview of such formulations and their advantages for VRPTW problems.

An example of a simple set partitioning formulation of ST-SR problems without temporal or ordering constraints is shown in Fig. 5. In the formulation, $Y_{a}$ is a set of routes for robot $a$ computed using the shortest path algorithm with resource constraints. $s_{y}^{a}$ is an indicator variable that assumes a value of 1 if robot $a$ is assigned route $y \in Y_{a}$ and 0 otherwise; $C_{y}^{a}$ is the cost robot $a$ incurs for performing route $y ; b_{y k}^{a}$ is a binary constant that is 1 if task $k$ is performed in route $y \in Y_{a}$ of robot $a$ and 0 otherwise.

A more complex formulation with cross-scheduling temporal and location dependencies, time windows, precedence and synchronization constraints is presented in Korsah [2011].

Stochastic allocations. In stochastic problems, it is assumed that a model of uncertainty is available. Stochastic ST-SR-TA:TW problems, like other stochastic 
$\operatorname{minimize} \sum_{a \in A} \sum_{y \in Y_{a}} C_{y}^{a} s_{y}^{a}$

subject to

(a) $\forall a \in A$

(b) $\forall k \in K$

(c) $\forall a \in A, y \in Y_{a} \quad s_{y}^{a} \in\{0,1\}$
$Y_{a}$ set of routes $y$ for $a$ and $C_{y}^{a}$ cost to $a$ of route $y$

Every robot gets at most 1 route

Each task is on 1 route

Indicator: route $y$ assigned to robot $a$

Figure 5: Set partitioning formulation for MRTA problems with no time windows and no capacity limits.

problems, are usually modeled as pure or mixed stochastic integer programs, or as Markov Decision Processes (MDPs) [Gendreau et al., 1996]. When modeled as stochastic integer programs [Ponda et al., 2012] they assume the form in Eq. 2 with the constraints shown in Fig. 4 or stochastic constraints [Shen et al., 2009]. In Eq. 2 the objective function is the expected reward, $\theta \in \Theta$ is the uncertainty model that is available to the robots, $U_{k}^{a}$ is the reward that robot a gets for doing task $k$, and $x_{k}^{a}$ the indicator of assignment of task $k$ to $a$.

$$
\operatorname{maximize} \mathbb{E}_{\theta}\left(\sum_{a \in A} \sum_{k \in K} U_{k}^{a} x_{k}^{a}\right)
$$

Examples of uncertainty models [Miao et al., 1991] include probability distributions for task arrival, robot travel time, task availability, and more.

Other stochastic formulations are used in the dynamic and stochastic VRPTW literature. For instance, [Bopardikar et al., 2014] studied a dynamic VRP problem in which demands (or tasks) with deterministic time constraints arrive randomly, and the goal is to maximize the fraction of demand met. In this work, vehicle motion is constrained and a reachability graph is used for navigation. In [Pavone et al., 2009] demand is also stochastic and there are time window constraints. In addition, demand disappears with known probabilities, which is used to model customer impatience. Both [Bopardikar et al., 2014] and [Pavone et al., 2009] analyze a number of requirements, such as bounds on the number of vehicles used and maximum number of tasks that can be missed. In order to prove theoretical properties both make some strong assumptions. For instance in [Pavone et al., 2009] all time windows need to have the same length.

An alternative way of modeling uncertainty uses MDPs. In [Dean et al., 1993, Beynier and Mouaddib, 2007] MDP states are locations in a map with obstacles, tasks, and robots. In [Beynier and Mouaddib, 2007] a state is a triplet representing the previously visited state, the amount of resources left, and the time window. The goal is to search for policies that maximize a value function over the states. Dolgov et al. [2007] poses the problem as a combinatorial resource scheduling problem with uncertainty, which can be easily extended to include locations, forming a MRTA problem.

Uncertainty models for ST-SR-TA:TW problems are, to the best of our 
knowledge, rarely explored in the MRTA literature, although stochastic planning could lead to practical gains in terms of producing sound and robust allocation policies for robots. Instead, it is more common to address stochasticity by model-free methods, such as reinforcement learning, or to deal with uncertainty by replanning during task execution.

\subsubsection{Soft Temporal Constraints}

Deterministic allocations. The only difference in this class of problems is the lack of hardness of the temporal constraints. In soft time window constraints for VRP, the goal is to find the best vehicle-task assignments that minimize the cost function $f(\cdot)$ of servicing some number of clients. In this case, the cost the sum of the fixed cost of operating the vehicles, the cost of operating the vehicles on the specific routes, and the penalty cost for arriving early or late to the clients on the routes [Taillard et al., 1997]. Penalty costs for arriving early may be different than for arriving late, (e.g. early arrival is a small penalty, late arrival is a larger penalty) and these may vary by domain. For instance, in complex mission planning for robots with communication constraints early and late arrivals are penalized equally because both can break the network connectivity [Ponda et al., 2010].

Stochastic allocations. Stochastic versions of ST-SR-TA:TW problems with soft constraints have an uncertainty model available like in the hard constraint case. However, they use soft windows and allow agents to gain value even when doing tasks outside their original time window. The objective is still to minimize a cost function (e.g distance or energy) or maximize a utility function, with the inclusion of some probability model; often these are probabilities of travel delay between tasks and therefore travel times, but could be specific to the tasks and affect other costs. For instance, Hsu et al. [2007] models the process of delivering perishable food, which affects inventory costs. Work in [Taş et al., 2013] models travel time delays with several distribution types, which change the service cost of operating a vehicle. The travel time probability directly affects whether the agent arrives early or late, which is why we frequently see stochastic formulations in soft time windows but no other kinds of preferred constraints.

\subsection{ST-SR-TA:SP - Single-Task robots, Single-Robot tasks, Time-Extended As- signments: Synchronization and Precedence}

Synchronization and precedence constraints can be formulated as in [Bredström and Rönnqvist, 2008] (Eq. 3 and Eq. 4). Let $k, k^{\prime} \in P$ where $P$ is a set of task pairs with precedence constraints, and $P^{s y n c} \subseteq P$ is the subset of tasks that have to start at the same time. Eq. 3 states that regardless of which robot(s) is assigned to tasks $k$ and $k^{\prime}$, task $k^{\prime}$ should start $\epsilon$ time units after the finish time of task $k$. If $\epsilon>0$ then $k, k^{\prime} \in P$ (Eq. 3), and if $\epsilon=0$ then $k, k^{\prime} \in P^{s y n c}$ (Eq. 4). $S_{k}$ and $F_{k}$ indicate again respectively the start and finish time of task $k$. 


$$
\begin{array}{rl}
\sum_{a \in A} S_{k^{\prime}} x_{k^{\prime}}^{a}-\sum_{a \in A} F_{k} x_{k}^{a}>\epsilon+M\left(1-o_{k k^{\prime}}^{a}\right) & \forall a \in A, k, k^{\prime} \in P, \epsilon>0 \\
\sum_{a \in A} S_{k^{\prime}} x_{k^{\prime}}^{a}-\sum_{a \in A} S_{k} x_{k}^{a}=0 & k, k^{\prime} \in P^{s y n c}
\end{array}
$$

ST-SR-TA:SP problems have received some attention in the MRTA literature. Barbulescu et al. [2010] interleave scheduling, task allocation, and execution of tasks with precedence and synchronization constraints. Other applications range from a music wall [Chopra and Egerstedt, 2012], where multiple tasks in different planar positions require simultaneous servicing, to assembling a structure with a team of robots [Heger et al., 2005], where there is an order in the assembly steps.

Luo et al. [2011] present a model for tasks with set precedence constraints that divides tasks into disjoint sets with strict ordering between the sets, and assumes that each robot can do at most one task per set. The model heavily constrains the type of allowable precedence graphs, but the algorithm proposed is proved to be sound and complete. A general model for allocation of tasks with any type of precedence constraint is presented in [McIntire et al., 2016].

Synchronization and precedence constraints can be used to model different types of temporal relationships between tasks. Precedence constraints typically are in the form that the start time of a task cannot occur earlier than the end time of any of its predecessors (end to start). Other, less used, precedence models include start to start, start to end, and end to end constraints [Lombardi and Milano, 2012]. Start to start constraints require that a task does not start until its precedents have started, the remaining types of precedence constraints follow a similar interpretation.

Sometimes executing a task precludes the execution of another. This type of problem is typically addressed at the planning stage, by enforcing precedence constraints between the tasks (e.g., [Olawsky and Gini, 1990]).

\subsection{ST-MR-TA:TW - Single-Task robots, Multi-Robot tasks, Time-Extended Assignments: Time Windows}

\subsubsection{Hard Temporal Constraints}

In ST-MR-TA:TW task allocation problems the robots need to work simultaneously on tasks as coalitions, while respecting the time window constraints. Coalition-based task allocation occurs when tasks cannot be executed by a single robot, or when task execution is more efficient when done by multiple robots [Shehory and Kraus, 1998, Vig and Adams, 2006]. In disaster rescue, for instance, fire fighters working in coalitions may extinguish the same number of fires earlier than if they had to work individually on each fire [Parker et al., 2016]. Moreover, in scenarios where the number of agents is limited, coalitionbased allocations may enable a higher task completion rate [Ramchurn et al., 2010b, Su et al., 2016]. 
Coalition formation, in general, requires dealing with two subproblems: coalition value computation and coalition structure generation [Sandholm et al., 1999]. The former is concerned with computing the expected utilities (or costs) of forming all possible coalitions, whereas the latter is concerned with partitioning the set of agents into exhaustive and disjoint groups that maximize the total utility. In MRTA, the coalition value is typically a combination of the utility gained and the coordination cost. Coalition size may be restricted by the physical constraints which limit the number of robots that can work simultaneously on the same task.

Let $2^{A}$ be the set of agent coalitions that may be formed with the agents in $A$ (i.e., all subsets of $A$ ) and $x_{k}^{c}$ be an indicator variable that takes the value of 1 if task $k$ is assigned to coalition $c \in 2^{A}$ and 0 otherwise. The coalition size is $|c|$ . For simplicity, we assume that all agents start their tours from an initial node 0 and finish at node $m+1$. Let $o_{k k^{\prime}}^{a}=1$ when agent $a$ visits task $k^{\prime}$ directly after $k$ and 0 otherwise. $o_{0 k}^{a}=1$ if $a$ visits $k$ at the very beginning of the route and 0 otherwise. Similarly, $o_{k(m+1)}^{a}=1$ denotes that $a$ visits task $k$ at the end of its route. An extra waiting time may be imposed after the task's earliest start time to form the coalition. When coalition work affects the task execution efficiency, the task duration should be computed accordingly [Ramchurn et al., 2010b].

ST-MR-TA:TW allocation problems can generally be formalized by the MILP in Fig. 6.

minimize or maximize $f($.

subject to
(a) $\forall k \in K$
$\sum_{c \in 2^{A}} x_{k}^{c} \leq 1$
(b) $\forall c \in 2^{A}, k \in K$
$\sum_{a \in c} x_{k}^{a}=|c| x_{k}^{c}$
Every task get at most one coalition.
(c) $\forall a \in A$
$\sum_{k \in K} o_{0 k}^{a}=1$
Coherence coalition-agent.
(d) $\forall a \in A$
$\sum_{k \in K} o_{k(m+1)}^{a}=1$
All robots $a$ start at initial location.
(e) $\forall a \in A, k^{\prime} \in K$
$\sum_{k \in K, k \neq k^{\prime}} o_{k k^{\prime}}^{a}$ $-\sum_{k^{\prime \prime} \in K, k^{\prime} \neq k^{\prime \prime}} o_{k^{\prime} k^{\prime \prime}}^{a}=0$
(f) $\forall a \in A, k, k^{\prime} \in K$
$S_{k}+D U R_{k}+T T_{k k^{\prime}}$
All robots $a$ end at final location.
(g) $\forall k \in K$
$-M *\left(1-o_{k k^{\prime}}^{a}\right) \leq S_{k^{\prime}}$
Routes are connected.
(h) $\forall k \in K$
$E S_{k} \leq S_{k} \leq L S_{k}$
Time between 2 consecutive tasks
(i) $\forall k \in K$
$E F_{k} \leq F_{k} \leq L F_{k}$
... allows for travel time.
$F_{k}-S_{k} \geq D U R_{k}$
(j) $\forall a \in A, k \in K \quad x_{k}^{a} \in\{0,1\}$
(k) $\forall c \in 2^{A}, k \in K \quad x_{k}^{c} \in\{0,1\}$
(l) $\forall a \in A, k, k^{\prime} \in K \quad o_{k k^{\prime}}^{a} \in\{0,1\}$
Start time in valid range.
Finish time in valid range.
Task time is long enough.
Indicator: robot $a$ does task $k$
Indicator: coalition $c$ does task $k$
Indicator: $a$ does task $k^{\prime}$ right after $k$.

Figure 6: Standard mixed integer linear formulation of the task allocation problem with single-task robots, multiple-robot tasks, and hard temporal constraints.

An alternative model for ST-MR-TA:TW problems is the set partitioning model shown earlier in Fig. 5. When cast as a set partitioning problem, a set 
of coalitions $C=\left\{c_{1}, \ldots, c_{|C|}\right\}$ corresponds to a set partition of $A$ if and only if the coalitions are exhaustive, i.e. $\bigcup_{c_{i} \in C} c_{i}=A$, and the elements of $C$ are pairwise disjoint, i.e., $\forall c_{i}, c_{j} \in C$ s.t. $i \neq j: c_{i} \cap c_{j}=\emptyset$. The solution to the set partition problem is a partition of A that maximizes the utility $u: S \rightarrow \mathbb{R}^{+}$. The NP-hard nature of problems in this class requires approximate solutions for practical coalition-based MRTA problems.

Certain side constraints, such as capability and resource constraints, are very important in this class of MRTA/TOC problems. Agents may have limited resources, especially in the case of small robots. For instance, in disaster rescue scenarios, fire trucks may need a certain amount of fuel to travel to a fire and a certain amount of water to extinguish it. Tasks might require coalitions of agents with specific capabilities. For instance, a fire might require a coalition of fire fighters, while police and ambulances can work together to dig out and carry survivors to refuge centers [Kitano and Satoshi, 2001, Parker et al., 2016].

MRTA researchers have proposed coalition-based frameworks for heterogeneous robots. Examples include ASyMTRe [Parker and Tang, 2006], an architecture to form coalitions for tasks that require tight robot coordination. The architecture uses a collection of schemas for perception and motor control, which are connected at run time, enabling the robots to share information as needed to complete the tasks. The architecture has been extended [Zhang and Parker, 2013a] to ensure that only feasible coalitions are formed. Efficient scheduling heuristics for coalitions are proposed in [Zhang and Parker, 2013b]. None of these works considers problems with time-extended assignments, they consider only instantaneous assignments.

Stochastic allocations. To the best of our knowledge, no literature addresses stochastic ST-MR-TA problems with either hard or soft constraints.

\subsubsection{Soft Temporal Constraints}

ST-MR-TA:TW with soft constraints assumes that multiple agents can work simultaneously on the same task and are allowed to violate some temporal constraints, with a penalty for the violation. The objective function in this case includes a temporal violation penalty:

$$
\underset{S \in 2^{A}}{\operatorname{argmax}} \sum_{c \in S} \sum_{k \in K} x_{k}^{c} U_{k}^{c} \pi_{k}^{S_{k}}
$$

where $S$ is a coalition structure, $U_{k}^{c}$ is coalition $c$ 's utility for performing task $k$ and $\pi_{k}^{S_{k}} \in[0,1]$ is the utility decay coefficient function for task $k$. This coefficient is set as $\pi_{k}^{S_{k}}=1$ when task $k$ is started and/or finished within the time window (i.e., $E S_{k} \leq t \leq L S_{k}$ and $S_{k}+D U R_{k} \leq L F_{k}$ ). Early and/or late task executions are penalized by setting $\pi_{k}^{S_{k}}$ to values in the range $[0,1]$. In particular, $\pi_{k}^{S_{k}}=0, \forall k \in K$ when $S_{k}+D U R_{k}>L S_{k}$ [Koes et al., 2005, Amador et al., 2014].

Most research on ST-MR-TA:TW problems with soft constraints is motivated by application areas such as urban search and rescue [Koes et al., 2005, 
Scerri et al., 2005], or law enforcement where police officers are assigned to crime events in a city [Amador et al., 2014]. In [Scerri et al., 2005] an expected utility model is used to allocate interdependent tasks. Late task executions are penalized by subtracting the delay cost from the total utility. The work subdivides large tasks into smaller subtasks that are linked with simultaneous execution interdependency, and coalitions of agents execute the smaller subtasks. The coalition formation problem is simplified by fixing the coalition size and reducing the number of allowed coalitions.

In [Koes et al., 2005] the task utility decays over time from the beginning of the mission and becomes zero by the mission deadline. Joint tasks can start only when all the robots are present and robot have to work on them for the entire duration. Likewise, in [Amador et al., 2014] the utility of tasks delayed beyond the soft deadline decays exponentially over time. The coalition value depends on the number of agents and is a function of the agents' fitness in performing a task.

\subsection{ST-MR-TA:SP - Single-Task robots, Multiple-Robot tasks, Time-Extended Assignments: Synchronization and Precedence}

ST-MR-TA:SP problems have received more limited attention than the STSR-TA:SP counterpart. Part of the reason might be the fact that current robotics applications do not use coalitions as a way to achieve tasks more efficiently.

In [Shehory and Kraus, 1998] tasks that require a set of capabilities are allocated to a set of robots with different types of capabilities. Robots form coalitions to perform tasks with precedence constraints. The work proposes greedy distributed set partitioning and set covering algorithms to give an approximate solution to the problem. In [Tang and Parker, 2007] the coalition formation problem is solved using ASyMTRe, and auctions are used for allocation of tasks with precedence constraints to coalitions. Coalitions bid through a coalition leader. The common theme among these works is that they do not handle synchronization constraints.

Sariel and Balch [2006] consider precedence and synchronization constraints together with dynamic allocation of tasks. Tasks require that a certain number of robots work on them. The goal is to minimize the makespan. The approach combines auctions with coalition maintenance, and employs recovery routines to deal with exogenous events. Both Jones et al. [2011] and Parker et al. [2016] handle temporal and precedence constraints in search and rescue domains. The precedence constraints are expressed in the form of deadline constraints.

Works that address Robocup Search and Rescue (e.g [Scerri et al., 2005, Parker et al., 2016]) often span both ST-MR-TA:TW and ST-MR-TW:SP, because robots can collect utilities on tasks only before tasks expire, and some

tasks can only be done after others have been completed (e.g blockades need to be removed before fires are extinguished). 
6.5. MT-SR-TA:TW - Multi-task robots, Single-robot tasks, Time-extended Assignments: Time Windows

This class of problems is no more common now than it was in [Gerkey and Matarić, 2004], but we can provide some additional context for multi-task robots. Gerkey's work likens the MT-SR problem to the ST-MR problem, using the same mathematical formulation for both problems but switching the role of tasks and agents in the formula. Multi-task robots do exist in real life.Examples include the mission-driven Mars rover Curiosity, which can perform tasks at a location, such as grasping and manipulating a close by object, and at the same time do tasks like take pictures. Reconnaissance drones may track objects and take pictures (a relatively easy task) or may need to track objects on the ground and drop packages (a more difficult task that includes more intense object manipulation). Hence, MT-SR problems are worth studying.

A multi-tasking robot can either preempt tasks or not; preempting tasks requires knowledge of task priorities and may require task rescheduling, whereas a simpler system with non-preemptive tasks may miss important tasks that arrive during execution. In preemptive cases where the robot was physically manipulating the environment, additional overhead time might be required to restore the robot's pose and the environment [Groth and Henrich, 2014]. In [Amador et al., 2014] tasks can be interrupted by higher priority tasks and resumed later with a penalty that decreases over time.

Additionally, robots must deal with failures; not only must the robot prioritize tasks, but it must decide (or have a plan for) what to do when the preempting task fails. Does the robot retry the failed task, move directly back to the preempted task, or drop into some kind of re-calibration or maintenance mode? Consider the Mars rover - if it runs into a rock or becomes stuck while navigating to a site where it has to perform chemical analysis, it should stop and get unstuck (or consult Earth-based humans for assistance), then return to navigation towards its earlier goal. If instead a piece of the rover's chemical analysis fails due to hardware problems, it should probably stop all analyses until it can relay its problems and receive solutions from Earth.

Very limited literature exists on multi-tasking robots; much of the work focuses instead on robots that have many tasks to do very close together temporally, for example a UAV that searches for objects, targets an object, and releases a bomb. Groth and Henrich [2014] discuss a multi-tasking robot with nearby tasks (taking pictures of people and finding objects, or taking pictures of walls and greeting humans) with preemption; the robot stops taking pictures of walls if a human is in the way, for example.

In general, the MT-SR problem, regardless of the type of time window, can be approached heuristically as a bin packing problem, where each robot is a bin and each task is assigned to a robot that has available capacity and resources to perform that task. Other approaches to scheduling tasks are inspired by operating systems, such as shortest job first and priority scheduling; however, because context switching is more time-demanding for robots than it is for processors, these methods may be highly suboptimal. 
6.6. MT-SR-TA:SP: Multiple-Task robots, Single-Robot tasks, Time-Extended Assignments: Synchronization and Precedence

Like its MT-SR-TA:TW counterpart, these types of problems have not received much attention in the MRTA literature. An exception is the work by Landén et al. [2012], which provides a distributed solution to a problem with precedence constraints. Complex tasks are modeled by a task specification tree, which specifies precedence and dependencies between tasks. A distributed constraint satisfaction solver is used to check constraint consistency. Task allocation is performed by a recursive search over feasible allocations.

\subsection{MT-MR-TA:TW - Multi-Task robots, Multi-Robot tasks, Time-Extended} Assignments: Time Windows

Multi-task robots and multi-robot task problems remain sparsely explored [Korsah et al., 2013, Gerkey and Matarić, 2004], even without considering temporal constraints. This class of problems can be modeled as an overlapping coalition formation problem [Chalkiadakis et al., 2010] combined with a routing and scheduling problem.

MT-MR-TA:TW problems can be decomposed into the following subproblems: (1) assigning coalitions to tasks, (2) assigning different coalitions to the same robot as long as no resource constraints are violated, and (3) assigning values to the start and finish times of tasks. Each of these subproblems is NP-hard.

Multi-task robots and multi-robot task problems can also be modeled as cooperative games with overlapping coalitions. In cooperative games with overlapping coalitions, agents can do more than one task at a time. This may lead robots to commit to a task assigned to more than one coalition. Overlapping coalitions have been used to model collaborative smartphone sensing in [Di et al., 2013]. In that work, smartphone users form overlapping networks, and an incentive function rewards users' contributions to different tasks. Unfortunately, finding the optimal overlapping coalition is NP-complete.

6.8. MT-MR-TA:SP - Multi-Task robots, Multi-Robot tasks, Time-Extended Assignments: Synchronization and Precedence

We could not find MRTA/TOC works for this part of the taxonomy.

\subsection{Summarizing the Taxonomy}

We summarize the literature in Table 2, by classifying according to our taxonomy papers published in the MRTA and related literatures between 2003 and 2016. In the table Det and Sto stand respectively for deterministic and stochastic. 


\begin{tabular}{|c|c|c|c|c|c|c|c|c|c|c|}
\hline Reference & ST & MT & SR & MR & TW & $\mathrm{SP}$ & $\mathrm{HC}$ & $\mathrm{SC}$ & Det & Sto \\
\hline [McIntire et al., 2016] & $\checkmark$ & & $\checkmark$ & & & $\checkmark$ & $\checkmark$ & & $\checkmark$ & \\
\hline [Luo et al., 2015] & $\checkmark$ & & $\checkmark$ & & $\checkmark$ & & $\checkmark$ & & $\checkmark$ & \\
\hline [Nunes and Gini, 2015] & $\checkmark$ & & $\checkmark$ & & $\checkmark$ & & $\checkmark$ & & $\checkmark$ & \\
\hline [Coltin and Veloso, 2014b] & $\checkmark$ & & $\checkmark$ & & $\checkmark$ & & $\checkmark$ & $\checkmark$ & $\checkmark$ & \\
\hline [Luo, 2014] & $\checkmark$ & & $\checkmark$ & & & $\checkmark$ & $\checkmark$ & & $\checkmark$ & \\
\hline [Gombolay et al., 2013] & $\checkmark$ & & $\checkmark$ & & $\checkmark$ & $\checkmark$ & $\checkmark$ & & $\checkmark$ & \\
\hline [Taş et al., 2013]* & $\checkmark$ & & $\checkmark$ & & $\checkmark$ & & & $\checkmark$ & & $\checkmark$ \\
\hline [Chopra and Egerstedt, 2012] & $\checkmark$ & & $\checkmark$ & & $\checkmark$ & & $\checkmark$ & & $\checkmark$ & \\
\hline [Korsah et al., 2012] & $\checkmark$ & & $\checkmark$ & & $\checkmark$ & $\checkmark$ & $\checkmark$ & & $\checkmark$ & \\
\hline [Barbulescu et al., 2010] & $\checkmark$ & & $\checkmark$ & & $\checkmark$ & $\checkmark$ & $\checkmark$ & & $\checkmark$ & \\
\hline [Ponda et al., 2010] & $\checkmark$ & & $\checkmark$ & & $\checkmark$ & & & $\checkmark$ & $\checkmark$ & \\
\hline [Pavone et al., 2009]* & $\checkmark$ & & $\checkmark$ & & $\checkmark$ & & $\checkmark$ & & & $\checkmark$ \\
\hline [Shah et al., 2009] & $\checkmark$ & & $\checkmark$ & & $\checkmark$ & $\checkmark$ & $\checkmark$ & & $\checkmark$ & \\
\hline [Bredström and Rönnqvist, 2008]* & $\checkmark$ & & $\checkmark$ & & $\checkmark$ & $\checkmark$ & $\checkmark$ & & $\checkmark$ & \\
\hline [Beynier and Mouaddib, 2007] & $\checkmark$ & & $\checkmark$ & & $\checkmark$ & & $\checkmark$ & & & $\checkmark$ \\
\hline [Melvin et al., 2007] & $\checkmark$ & & $\checkmark$ & & $\checkmark$ & & $\checkmark$ & & $\checkmark$ & \\
\hline [Ando and Taniguchi, 2006] ${ }^{*}$ & $\checkmark$ & & $\checkmark$ & & $\checkmark$ & & $\checkmark$ & & & $\checkmark$ \\
\hline [Heger et al., 2005] & $\checkmark$ & & $\checkmark$ & & & $\checkmark$ & $\checkmark$ & & $\checkmark$ & \\
\hline [Alighanbari et al., 2003] & $\checkmark$ & & $\checkmark$ & & & $\checkmark$ & $\checkmark$ & & $\checkmark$ & \\
\hline [Su et al., 2016] & $\checkmark$ & & & $\checkmark$ & $\checkmark$ & & $\checkmark$ & & $\checkmark$ & \\
\hline [Pujol-Gonzalez et al., 2015] & $\checkmark$ & & & $\checkmark$ & $\checkmark$ & $\checkmark$ & $\checkmark$ & & $\checkmark$ & \\
\hline [Parker et al., 2016] & $\checkmark$ & & & $\checkmark$ & $\checkmark$ & $\checkmark$ & & $\checkmark$ & $\checkmark$ & \\
\hline [Amador et al., 2014] & $\checkmark$ & & & $\checkmark$ & $\checkmark$ & & & $\checkmark$ & $\checkmark$ & \\
\hline [Jones et al., 2011] & $\checkmark$ & & & $\checkmark$ & & $\checkmark$ & $\checkmark$ & & $\checkmark$ & \\
\hline [Ramchurn et al., 2010b] & $\checkmark$ & & & $\checkmark$ & $\checkmark$ & & $\checkmark$ & & $\checkmark$ & \\
\hline [Tang and Parker, 2007] & $\checkmark$ & & & $\checkmark$ & & $\checkmark$ & $\checkmark$ & & $\checkmark$ & \\
\hline [Sariel and Balch, 2006] & $\checkmark$ & & & $\checkmark$ & & $\checkmark$ & $\checkmark$ & & $\checkmark$ & \\
\hline [Scerri et al., 2005] & $\checkmark$ & & & $\checkmark$ & $\checkmark$ & $\checkmark$ & & $\checkmark$ & $\checkmark$ & \\
\hline [Koes et al., 2005] & $\checkmark$ & & & $\checkmark$ & $\checkmark$ & $\checkmark$ & & $\checkmark$ & $\checkmark$ & \\
\hline [Landén et al., 2012] & & $\checkmark$ & $\checkmark$ & & & $\checkmark$ & $\checkmark$ & & I & \\
\hline
\end{tabular}

Table 2: Selected papers from each category of the taxonomy. Papers with a ${ }^{*}$ symbol are not MRTA papers, but are included for completeness.

\section{Dynamic Task Release and Execution}

Execution of tasks in MRTA/TOC problems vary according to the dynamics considered. Dynamics may be due to faulty robots, changes in estimated cost due to uncertainties, changes in task definitions, online arrival of tasks, addition of robots to the team, and other changes made by external agents [Sariel-Talay et al., 2009]. While the execution aspect is outside the task allocation scope, the planning-execution-replanning of tasks forms a planning loop that is usually addressed at once in dynamic domains. Here we consider dynamics caused by task arrival and during task execution separately. 
Some dynamics are caused by the arrival of tasks over time without further knowledge of future tasks. Usually when a new task arrives there is already an existing allocation for previously scheduled tasks that have not yet been executed. Thus, replanning occurs at task arrivals, while robots are executing previously assigned tasks [Cordeau and Laporte, 2007]. In [Nunes and Gini, 2015] both deterministic and dynamic task arrivals are considered, assuming the robots have perfect knowledge of the map where tasks appear. In contrast, problems usually defined as online pickup and delivery problems or dial-a-ride include not only online arrival of tasks but other uncertain events, such as vehicle breakdowns and delays [Cordeau and Laporte, 2007]. Recent examples of online pickup and delivery consider transfers, in addition to the arrival of tasks with hard temporal constraints [Bouros et al., 2011, Coltin and Veloso, 2014a,b].

The dynamics that occur during plan execution [Block et al., 2006, SarielTalay et al., 2009, Shah et al., 2009] are very important for the practical use of robots, because execution can fail due to many reasons and replanning is essential to maintain some level of efficiency. In [Barbulescu et al., 2010] dynamics

during execution are created by unexpected events and changes in costs and constraints; in [Ponda, 2012] dynamics are caused by breaks in communication links, which may cause conflicting assignments, as more than one robot could be assigned the same task. In [Usug and Sariel-Talay, 2011] temporary failures are considered, such as obstacles, which can be overcome by replanning.

\section{Typical Solution Approaches}

So far, we have proposed a taxonomy for MRTA/TOC problems; now we discuss the most popular solutions and how to map these to our taxonomy. Here we simply divide the methods into centralized vs. decentralized. Centralized methods are further separated into exact and approximate methods, while decentralized methods are grouped into distributed constraint-based and market-based according to nature of the proposed solutions.

\subsection{Centralized Solutions}

Centralized methods rely on a central controller that allocates tasks to robots. The autonomy of the robots in pure centralized methods is limited or non-existent, as they solely execute the dispatched orders and do not make decisions on what tasks to do.

MRTA/TOC is intractable for a non-trivial number of robots and tasks. Optimal centralized solutions are intractable because they need to evaluate a large number of candidate solutions in order to guarantee optimality. Thus, the focus of MRTA/TOC solutions is largely on approximation and heuristic solution methods. We discuss some of the common centralized exact and heuristic methods next. 


\subsubsection{Exact Solutions}

Exact solutions are optimal, but their computation time is impractical for realistic robotics applications. The most naive way to search for such solutions is to exhaustively search for all possible allocations that do not violate the temporal constraints. This is, however, intractable, because an exhaustive search leads to worst-case $O(|K| !)^{|A|}$ complexity for $|K|$ tasks and $|A|$ robots. We have to search through all the possible sequences of tasks and all possible allocations of tasks to robots, and in addition to all feasible assignments of times to tasks.

Optimal solutions can be more efficiently computed using Branch-and-Bound (B\&B) [Clausen, 1997] and its variants: Branch-and-Cut [Bard et al., 2002, Ropke et al., 2007], Branch-and-Price [Barnhart et al., 1998, Dohn et al., 2009, Feillet, 2010, Korsah et al., 2012], and Branch-Price-and-Cut [Barnhart et al., 2000]. B\&B searches the state space of candidate solutions represented as a tree

and uses upper and lower bounds of the optimal solution to prune the branches of the search tree that have costs higher than the computed lower bounds. Among the variants, Branch-Price-and-Cut is becoming popular in VRPTW [Ropke and Cordeau, 2009, Desaulniers, 2010, Archetti et al., 2011, Bettinelli et al., 2011]. However, as far as we know it has not been used in MRTA/TOC problems.

Exact methods often use tools such as CPLEX [ILOG, 2006], Gurobi [Gurobi Optimization, 2014], ABACUS [Jünger and Thienel, 2000], lp_solve [Berkelaar et al., 2004] or other tools to build and solve the underlying MILP formulations. MILP-based formulations and solutions have been predominantly used in ST-SR-TA:TW problems (e.g., [Alighanbari et al., 2003, Korsah et al., 2012], but these models have also been used in other parts of the taxonomy (e.g. STMR-TA:TW [Koes et al., 2005, Ramchurn et al., 2010b]).

\subsubsection{Approximate and Heuristic Solutions}

To reduce computation time, MILP-based heuristics are used to find approximate partial allocations while searching the state-space tree. These methods do not provide theoretical guarantees, but in some cases (e.g. [Gombolay et al., 2013]) they experimentally achieve results that are only $10 \%$ away from the optimal value (makespan).

Another way to gain computational efficiency is to use metaheuristic approaches. Metaheuristics are algorithmic templates that approximately solve hard combinatorial optimization problems. Unlike other combinatorial optimization algorithms, metaheuristics may allow lower quality solutions in the search process to escape local optima, and often embed off-the-shelf heuristics to solve the problem [Bräysy and Gendreau, 2005b, Vidal et al., 2013].

Metaheuristic approaches to VRPTW, TOPTW, and related routing and scheduling problems have been shown to outperform many other methods (e.g. construction heuristics and local search) for standard benchmarks [Bräysy and Gendreau, 2005b, Hu and Lim, 2014]. Recent trends in the metaheuristic literature seek to reduce the computation time and improve the solution quality by using parallelization and hybridization of different heuristics and exact 
techniques (e.g., Mitiche et al. [2015a]). However, metaheuristic parameters remain hard to tune [Bräysy and Gendreau, 2005b, Birattari, 2009, Mitiche et al., 2015b].

\subsection{Decentralized Solutions}

Decentralized approaches vary widely; a detailed categorization is outside the scope of this paper. Here we focus on (1) distributed constraint optimization and (2) market- and negotiation-based algorithms since these have received a great deal of attention in the MRTA community.

\subsubsection{Distributed Constraint (DCOP)-Based Methods}

MRTA/TOC problems can be modeled as a Distributed Constraint Optimization Problem (DCOP) [Maheswaran et al., 2004] and solved using DCOP methods. Solving DCOP exactly is NP-hard and impractical even for unconstrained MRTA problems [Junges and Bazzan, 2008]. Thus, approximate methods such as Max-Sum have been used for task allocation in sensor networks [Farinelli et al., 2014] and in RoboCup Rescue [Ramchurn et al., 2010a, PujolGonzalez et al., 2015].

Ramchurn et al. [2010a] proposed the Fast Max-Sum algorithm, which was shown to be robust in situations where the number of tasks is dynamic; the approach reduced the computation time, number and size of messages sent compared to Max-Sum, but it is still exponential. The computation overhead in dynamic environments is reduced in [Macarthur et al., 2011] by using online domain pruning and branch-and-bound. When the constraints are expressed as Tractable Higher Order Potentials the computation time can be reduced to polynomial [Pujol-Gonzalez et al., 2015].

Another approximation method is LA-DCOP [Scerri et al., 2005, Farinelli et al., 2006], which uses token passing [Xu et al., 2005] as follows: when an agent perceives a task, it creates a token for it. It can decide to do the task or pass the token to a randomly chosen agent. This tends to guide the search quickly towards a greedy solution, which is reasonable for ST-SR-TA:TW problems.

In [Ferreira et al., 2008] LA-DCOP and Swarm-GAP are compared in RoboCup settings. In Swarm-GAP an agent chooses a task according to a probability that depends on the stimulus generated by the task and the agent's threshold. Results show that both DCOP approaches behave similarly, and both perform better than a greedy task allocation. Their approach works for ST-MR-SC problems, where agents are allowed to arrive late to tasks. To facilitate comparing the performance of DCOP algorithms, RMASBench, a system that provides a library of state-of-the-art solvers for DCOP and for comparing them, has been created in [Kleiner et al., 2013].

\subsubsection{Market and Negotiation-Based Methods}

Among the decentralized algorithms, sequential auction- and negotiationbased algorithms [e.g. Sariel-Talay et al., 2009, Nanjanath and Gini, 2010, Ponda et al., 2010, Nunes and Gini, 2015] are more prevalent than other methods. 
Sequential auction algorithms produce solutions that are two away from optimal in the worst-case in both single-item [Lagoudakis et al., 2004] and multi-item auctions [Choi et al., 2009]. This, together with the ease of implementation and extension to dynamic scenarios and robust execution [Nanjanath and Gini, 2010] makes sequential auctions an attractive solution. However, the greedy nature of sequential auctions and the complex structure of most MRTA/TOC problems cause the addition of temporal constraints to auction algorithms to produce suboptimal solutions [Nunes et al., 2012]. Temporal modeling and balancing between temporal- and distance-based objectives can help auctions perform better [Nunes and Gini, 2015]. In [Amador et al., 2014] Fisher markets are used for dynamic ST-MR-SC problems, where tasks can be interrupted for a penalty.

Auctions distribute the computation to individual agents but require communication to share bids and results. To reduce the need for communication, several approaches use consensus algorithms [Zavlanos et al., 2008, Choi et al., 2009, Ponda, 2012], where each agent determines independently which tasks it should do. An equilibrium is reached by iteratively sharing information with neighbors and re-allocating tasks if needed. [Godoy and Gini, 2012] extended the Consensus Based Bundle Algorithm (CBBA) [Choi et al., 2009] to optimize the number of completed tasks for tasks with temporal constraints. Emergent task allocation [Atay and Bayazit, 2003], distributes the computation of task allocation for a surveillance task to individual robots, by sharing intentions and directives with 1-hop away neighbors. The method has been shown to converge to the optimal solution as the number of iterations of information sharing increases.

There are other decentralized approaches to task allocation that are not market- or DCOP-based. For instance, [Chapman et al., 2010] formulated STMR MRTA as a stochastic game and used overlapping potential games to approximate an optimal solution. Their approach is robust to restricted agent communication and observation range.

Swarm-based approaches have been proposed for various tasks, such as foraging, where robots need to find food and bring it to the nest [Lerman et al., 2006, Brutschy et al., 2014] or where swarms of robots are allocated different monitoring tasks without any communication among the robots [Berman et al., 2009]. Swarm methods often work well but do not have theoretical guarantees.

Despite the development of many decentralized methods for MRTA/TOC problems, very limited work offers theoretical analysis of the quality of these solutions. There is a need for theoretical performance bounds for both centralized and decentralized heuristics for the MRTA/TOC problem.

\section{Summary, Open Issues, and Directions for Future Research}

Problems that consider temporal and ordering constraints relate to many well studied problems, such as vehicle routing, job-shop scheduling, and multirobot task allocation. A large portion of the literature in MRTA/TOC focuses 
on ST-SR-TA:TW problems with hard constraints, some address the soft constraint version of this class of problem; however, the literature is sparser for other classes of problems that consider multi-task robots and multi-robot tasks.

\subsection{Summary}

We examined the MRTA/TOC literature related to problems where tasks have constraints on where, when, and the order or time in which they have to be performed. We extended a previous taxonomy and added a classification that separates the literature according to the use of time windows or synchronization/precedence constraints. Where appropriate, we gave a generic mathematical formulation of problems both in deterministic and stochastic cases, and offered an account of some common execution dynamics. We briefly discussed the methods applied to the problems in our taxonomy, and split solutions into centralized and decentralized approaches. In addition, our work drew parallels between multi-robot task allocation with temporal and ordering constraints with other areas of research, and throughout the paper we discussed models and solutions coming from these areas.

\subsection{Open Issues and Future Research}

There are several open issues, which we did not fully address here. Progress in the following topics would greatly advance research in MRTA/TOC: (1) study of theoretical guarantees of approximate solutions, (2) richer and more complex temporal models with provably good and efficient algorithms, (3) models and algorithms for stochastic MRTA/TOC problems, (4) models and algorithms for task allocation to multi-task robots, (5) studies on the effects of time scales and time sensitivity in MRTA/TOC problems and (6) the development of a research platform to make software and data available to researchers.

There is a need for work on theoretical guarantees for heuristic schedulers developed for MRTA/TOC problems. The NP-complete nature of the problem and the need for relatively fast planners has generated many heuristics. However, such heuristics typically lack performance guarantees, which can be crucial for safety critical systems, to ensure that robots work effectively even in the worst possible scenarios.

More work needs to be done to address more complex temporal constraint types, such as disjunctive temporal models. The literature could also benefit from work that combines soft and hard time windows, and precedence with synchronization constraints. A mix of these constraints might produce more expressive models for a larger set of real-world problems.

Research in stochastic MRTA/TOC problems is still very sparse. The development of MRTA methods that take advantage of simulation and stochastic models to better plan under uncertainty is an endeavor worth pursuing because robots often operate in uncertain environments. Important research questions can be asked here; for example, in an uncertain environment is it more beneficial to build a complex model that incorporates uncertainty, or is it enough to build less well-informed plans and replan as often as needed to quickly react to unexpected events? 
The challenge of allocating tasks to multi-task robots, which are robots that can perform more than one task at a time, remains open. As we can imagine this is difficult for many existing robots because they lack the necessary actuators. The lack of literature might also be due to the lack of practical applications of multi-task robots. We are not aware of any practical problems that strictly requires robots to perform multiple tasks concurrently; one example of such problems could be in military domains where a drone robot could be required to strike a target while at the same tracking other targets in nearby areas.

Another interesting, yet not so explored topic, regards time scales and sensitivity. Robots that move and navigate in an environment can be on a short time scale, as in exploring a building in hours, or a large time scale of exploring a planet for years. Even for a single robot, tasks can have different time sensitivities; some tasks may have short horizons with hard time constraints, whereas others may have long time horizons with soft time windows. For instance, the Mars rover Curiosity has periodic tasks that occur every day for years (data upload), constantly running tasks (temperature regulation), and sporadic tasks (chemical analysis of collected material and drilling). Each of these tasks have different time sensitivities; for example, data upload needs to occur when the receiving orbiters are within view of the rover. Temperature regulation requires constant vigilance, and drilling can be postponed, but needs to occur when the rover is within reach of the material. Chemical analysis in the rover's internal chambers can occur regardless of location. This single robot has tasks with hard time windows, soft time windows, and different time sensitivities. Considering tasks in terms of time scales and task sensitivities in the same robotic system thus holds value for any researcher interested in real world problems.

Lastly, we are concerned with the public availability of research data and methods. We advocate for a computational infrastructure for MRTA problems (in general, or in particular problems with temporal and ordering constraints). A tool identical to the Computational Infrastructure for Operations Research COIN-OR [2015]) could greatly benefit MRTA researchers. COIN-OR is an open source software project in which many operations research algorithms are implemented and maintained by scholars in the area. That in combination with datasets would help researchers verify their results on publicly available data and methods, allowing for richer comparisons among methods.

Acknowledgment: Partial support is gratefully acknowledged from NSF grant CNF-1531330 and IIS-1208413.

\section{References}

Alighanbari, M., Kuwata, Y., How, J. P., Jun. 2003. Coordination and control of multiple UAVs with timing constraints and loitering. In: American Control Conf. pp. 5311-5316.

Allahverdi, A., Ng, C., Cheng, T., Kovalyov, M. Y., 2008. A survey of scheduling problems with setup times or costs. European Journal of Operational Research 187 (3), $985-1032$. 
Allen, J. F., Nov. 1983. Maintaining knowledge about temporal intervals. Comm. of the ACM 26 (11), 832-843.

Amador, S., Okamoto, S., Zivan, R., 2014. Dynamic multi-agent task allocation with spatial and temporal constraints. In: Proc. AAAI Conf. on Artificial Intelligence. pp. 1384-1390.

Ando, N., Taniguchi, E., 2006. Travel time reliability in vehicle routing and scheduling with time windows. Networks and Spatial Economics 6 (3-4), 293311.

Archetti, C., Bouchard, M., Desaulniers, G., 2011. Enhanced branch and price and cut for vehicle routing with split deliveries and time windows. Transportation Science 45 (3), 285-298.

Atay, N., Bayazit, B., 2003. Emergent task allocation for mobile robots. In: Robotics: Science and Systems (RSS).

Balas, E., Simonetti, N., Vazacopoulos, A., 2008. Job shop scheduling with setup times, deadlines and precedence constraints. Journal of Scheduling 11 (4), $253-262$.

Barbulescu, L., Rubinstein, Z. B., Smith, S. F., Zimmerman, T. L., 2010. Distributed coordination of mobile agent teams: the advantage of planning ahead. In: Int'l Conf. on Autonomous Agents and Multi-Agent Systems. pp. $1331-1338$.

Bard, J. F., Kontoravdis, G., Yu, G., 2002. A branch-and-cut procedure for the vehicle routing problem with time windows. Transportation Science 36 (2), $250-269$.

Barnhart, C., Hane, C. A., Vance, P. H., 2000. Using branch-and-price-and-cut to solve origin-destination integer multicommodity flow problems. Operations Research 48 (2), 318-326.

Barnhart, C., Johnson, E. L., Nemhauser, G. L., Savelsbergh, M. W. P., Vance, P. H., 1998. Branch-and-price: Column generation for solving huge integer programs. Operations Research 46 (3), 316-329.

Beccari, G., Caselli, S., Reggiani, M., Zanichelli, F., 1999. Rate modulation of soft real-time tasks in autonomous robot control systems. In: Proc. 11th Euromicro Conference on Real-Time Systems. IEEE, pp. 21-28.

Beck, L. C., Refalo, P., 2003. A hybrid approach to scheduling with earliness and tardiness costs. Annals of Operations Research 118 (1-4), 49-71.

Berkelaar, M., Eikland, K., Notebaert, P., 2004. lp_solve 5.5, open source (mixed-integer) linear programming system.

URL http://lpsolve.sourceforge.net/5.5/ 
Berman, S., Halasz, A., Hsieh, M., Kumar, V., 2009. Optimized stochastic policies for task allocation in swarms of robots. IEEE Trans. on Robotics 25, $927-937$.

Bernat, G., Burns, A., Liamosi, A., 2001. Weakly hard real-time systems. IEEE Trans. on Computers 50 (4), 308-321.

Bettinelli, A., Ceselli, A., Righini, G., 2011. A branch-and-cut-and-price algorithm for the multi-depot heterogeneous vehicle routing problem with time windows. Transportation Research Part C: Emerging Technologies 19 (5), 723-740.

Beynier, A., Mouaddib, A.-I., 2007. Decentralized Markov Decision Processes for handling temporal and resource constraints in a multiple robot system. In: Alami, R., Chatila, R., Asama, H. (Eds.), Distributed Autonomous Robotic Systems 6. Springer Japan, pp. 191-200.

Birattari, M., 2009. Tuning Metaheuristics: A Machine Learning Perspective. Springer.

Bistarelli, S., Pini, M., Rossi, F., Venable, K., 2007. Bipolar preference problems: Framework, properties and solving techniques. In: Azevedo, F., Barahona, P., Fages, F., Rossi, F. (Eds.), Recent Advances in Constraints. Vol. 4651 of Lecture Notes in Computer Science. Springer, pp. 78-92.

Block, S. A., Wehowsky, A. F., Williams, B. C., 2006. Robust execution on contingent, temporally flexible plans. In: Proc. AAAI Conf. on Artificial Intelligence. pp. 802-808.

Boerkoel, J. C., Durfee, E. H., 2012. A distributed approach to summarizing spaces of multiagent schedules. In: Proc. AAAI Conf. on Artificial Intelligence. pp. 1742-1748.

Boerkoel, J. C., Durfee, E. H., 2013. Decoupling the multiagent disjunctive temporal problem. In: Int'l Conf. on Autonomous Agents and Multi-Agent Systems. pp. 1145-1146.

Boerkoel, J. C., Planken, L. R., 2012. Distributed algorithms for incrementally maintaining multiagent simple temporal networks. In: Int'l Conf. on Autonomous Agents and Multi-Agent Systems. pp. 216-235.

Bopardikar, S., Smith, S., Bullo, F., 2014. On dynamic vehicle routing with time constraints. IEEE Trans. on Robotics 30 (6), 1524-1532.

Borning, A., Freeman-Benson, B., Wilson, M., 1992. Constraint hierarchies. Lisp Symb. Comput. 5 (3), 223-270.

Bouros, P., Sacharidis, D., Dalamagas, T., Sellis, T., 2011. Dynamic pickup and delivery with transfers. In: Proc. 12th Int'l Conf. on Advances in Spatial and Temporal Databases (SSTD'11). pp. 112-129. 
Bräysy, O., Gendreau, M., 2005a. Vehicle routing problem with time windows, part I: Route construction and local search algorithms. Transportation Science 39 (1), 104-118.

Bräysy, O., Gendreau, M., 2005b. Vehicle routing problem with time windows, part II: Metaheuristics. Transportation Science 39 (1), 119-139.

Bredström, D., Rönnqvist, M., 2008. Combined vehicle routing and scheduling with temporal precedence and synchronization constraints. European Journal of Operational Research 191 (1), 19-31.

Brucker, P., Drexl, A., Möhring, R. H., Neumann, K., Pesch, E., 1999. Resourceconstrained project scheduling: Notation, classification, models, and methods. European Journal of Operational Research 112 (1), 3-41.

Brutschy, A., Pini, G., Pinciroli, C., anf Marco Dorigo, M. B., 2014. Selforganized task allocation to sequentially interdependent tasks in swarm robotics. Journal of Autonomous Agents and Multi-agent Systems 28 (1), $101-125$.

Caccamo, M., Buttazzo, G., 1997. Exploiting skips in periodic tasks for enhancing aperiodic responsiveness. In: Proc. IEEE Real-Time Systems Symposium. pp. 330-339.

Carrabs F., Cordeau J.-F., L. G., 2007. Variable neighborhood search for the pickup and delivery traveling salesman problem with LIFO loading. INFORMS Journal on Computing 19 (4), 618-632.

Cesta, A., Oddi, A., May 1996. Gaining efficiency and flexibility in the simple temporal problem. In: 3rd Int'l Workshop on Temporal Representation and Reasoning. pp. $45-50$.

Cesta, A., Oddi, A., Smith, S., 1999. An iterative sampling procedure for resource constrained project scheduling with time windows. In: Proc. Int'l Joint Conf. on Artificial intelligence. pp. 1022-1029.

Cesta, A., Oddi, A., Smith, S., 2000. A constraint-based method for project scheduling with time windows. Journal of Heuristics 8 (1), 109-136.

Chalkiadakis, G., Elkind, E., Markakis, E., Polukarov, M., Jennings, N. R., 2010. Cooperative games with overlapping coalitions. Journal of Artificial Intelligence Research 39 (1), 179-216.

Chao, I.-M., Golden, B. L., Wasil, E. A., 1996. The team orienteering problem. European Journal of Operational Research 88 (3), 464-474.

Chapman, A. C., Micillo, R. A., Kota, R., Jennings, N. R., 2010. Decentralized dynamic task allocation using overlapping potential games. The Computer Journal 53 (9), 1462-1477. 
Cheng, P., Keller, J., Kumar, V., Sep. 2008. Time-optimal UAV trajectory planning for 3D urban structure coverage. In: Proc. IEEE/RSJ Int. Conf. on Intelligent Robots and Systems. pp. 2750-2757.

Choi, H.-L., Brunet, L., How, J., 2009. Consensus-based decentralized auctions for robust task allocation. IEEE Trans. on Robotics 25 (4), 912-926.

Chopra, S., Egerstedt, M. B., 2012. Multi-robot routing for servicing spatiotemporal requests: A musically inspired problem. In: IFAC Conf. on Analysis and Design of Hybrid Systems. pp. 319-324.

Clausen, J., 1997. Branch and bound algorithms-principles and examples. Parallel Computing in Optimization, 239-267.

COIN-OR, 2015. COmputational INfrastructure for Operations Research. URL http://www. coin-or.org

Coles, A., Coles, A., Fox, M., Long, D., 2009. Incremental constraint-posting algorithms in interleaved planning and scheduling. In: Proc. Workshop on Constraint Satisfaction Techniques for Planning and Scheduling (COPLAS) at ICAPS. pp. 1-8.

Collins, J., Bilot, C., Gini, M., Mobasher, B., 2000. Mixed-initiative decision support in agent-based automated contracting. In: Proc. Int'l Conf, on Autonomous Agents. pp. 247-254.

Collins, J., Gini, M., 2006. MAGNET: A multi-agent system using auctions with temporal and precedence constraints. In: Chaib-draa, B., Müller, J. (Eds.), Multiagent based Supply Chain Management. Vol. 28. Springer, pp. 273-314.

Colorni, A., Righini, G., 2001. Modeling and optimizing dynamic dial-a-ride problems. International Transactions in Operational Research 8 (2), 155-166.

Coltin, B., Veloso, M., 2014a. Online pickup and delivery planning with transfers for mobile robots. In: Proc. IEEE Int'l Conf. on Robotics and Automation. pp. 5786-5791.

Coltin, B., Veloso, M. M., 2014b. Scheduling for transfers in pickup and delivery problems with very large neighborhood search. In: Proc. AAAI Conf. on Artificial Intelligence. pp. 2250-2256.

Cordeau, J.-F., Laporte, G., 2007. The dial-a-ride problem: models and algorithms. Annals of Operations Research 153 (1), 29-46.

Dantzig, G. B., Ramser, J. H., 1959. The truck dispatching problem. Management Science 6 (1), 80-91.

Davis, R. I., Burns, A., 2011. A survey of hard real-time scheduling for multiprocessor systems. ACM Comput. Surv. 43 (4), 35:1-35:44. 
Dean, T., Kaelbling, L. P., Kirman, J., Nicholson, A., 1993. Planning with deadlines in stochastic domains. In: Proc. AAAI Conf. on Artificial Intelligence. pp. $574-579$.

Dechter, R., Meiri, I., Pearl, J., 1991. Temporal constraint networks. Artificial Intelligence 49 (1-3), 61-95.

Desaulniers, G., 2010. Branch-and-price-and-cut for the split-delivery vehicle routing problem with time windows. Operations research 58 (1), 179-192.

Desrochers, M., Lenstra, J. K., Savelsbergh, M. W., Soumis, F., 1988. Vehicle routing with time windows: Optimization and approximation. Vehicle Routing: Methods and Studies 16, 65-84.

Di, B., Wang, T., Song, L., Han, Z., 2013. Incentive mechanism for collaborative smartphone sensing using overlapping coalition formation games. In: Porc. IEEE Global Communications Conf. (GLOBECOM). pp. 1705-1710.

Dohn, A., Kolind, E., Clausen, J., 2009. The manpower allocation problem with time windows and job-teaming constraints: A branch-and-price approach. Computers and Operations Research 36, 1145-1157.

Dolgov, D. A., James, M. R., Samples, M. E., 2007. Combinatorial resource scheduling for multiagent MDPs. In: Int'l Conf. on Autonomous Agents and Multi-Agent Systems. pp. 657-664.

Domshlak, C., Prestwich, S., Rossi, F., Venable, K., Walsh, T., 2006. Hard and soft constraints for reasoning about qualitative conditional preferences. Journal of Heuristics 12 (4-5), 263-285.

Dondo, R., Cerdá, J., 2007. A cluster-based optimization approach for the multidepot heterogeneous fleet vehicle routing problem with time windows. European Journal of Operational Research 176 (3), 1478-1507.

Fakcharoenphol, J., Harrelson, C., Rao, S., 2007. The K-traveling repairmen problem. ACM Trans. Algorithms 3 (4), Article No. 40.

Fang, C., Yu, P., Williams, B. C., 2014. Chance-constrained probabilistic simple temporal problems. In: Proc. AAAI Conf. on Artificial Intelligence. pp. 22642270 .

Farinelli, A., Iocchi, L., Nardi, D., Ziparo, V., 2006. Assignment of dynamically perceived tasks by token passing in multirobot systems. Proceedings of the IEEE 94 (7), 1271-1288.

Farinelli, A., Rogers, A., Jennings, N., 2014. Agent-based decentralised coordination for sensor networks using the max-sum algorithm. Journal of Autonomous Agents and Multi-agent Systems 28 (3), 337-380.

Feillet, D., 2010. A tutorial on column generation and branch-and-price for vehicle routing problems. 4OR-Q J. Operation Research 8 (4), 407-424. 
Ferreira, P., Boffo, F., Bazzan, A., 2008. Using Swarm-GAP for distributed task allocation in complex scenarios. In: Jamali, N., Scerri, P., Sugawara, T. (Eds.), Massively Multi-Agent Technology. Vol. 5043 of Lecture Notes in Computer Science. Springer Berlin / Heidelberg, pp. 107-121.

Gendreau, M., Laporte, G., Séguin, R., 1996. Stochastic vehicle routing. European Journal of Operational Research 88 (1), 3-12.

Gerkey, B. P., Matarić, M. J., 2004. A formal analysis and taxonomy of task allocation in multi-robot systems. The International Journal of Robotics Research 23 (9), 939-954.

Godoy, J., Gini, M., 2012. Task allocation for spatially and temporally distributed tasks. In: Proc. of the Int'l Conf. on Intelligent Autonomous Systems. pp. 603-612.

Gombolay, M., Wilcox, R., Shah, J., 2013. Fast scheduling of multi-robot teams with temporospatial constraints. In: Robotics: Science and Systems (RSS). Berlin, Germany, pp. 49-56.

Graham, R. L., Lawler, E. L., Lenstra, J. K., Kan, A. R., 1979. Optimization and approximation in deterministic sequencing and scheduling: a survey. Annals of Discrete Mathematics 5, 287-326.

Groth, C., Henrich, D., 2014. Single-shot learning and scheduled execution of behaviors for a robotic manipulator. In: Proc. 41st Int'l Symposium on Robotics (ISR). pp. 1-6.

Gurobi Optimization, I., 2014. Gurobi optimizer reference manual. URL http://www.gurobi.com

Hasgül, S., Saricicek, I., Ozkan, M., Parlaktuna, O., 2009. Project-oriented task scheduling for mobile robot team. Journal of Intelligent Manufacturing 20 (2), $151-158$.

Heap, B., Pagnucco, M., 2014. Minimising undesired task costs in multi-robot task allocation problems with in-schedule dependencies. In: Proc. AAAI Conf. on Artificial Intelligence. pp. 2542-2548.

Heger, F. W., Hiatt, L. M., Sellner, B., Simmons, R., Singh, S., 2005. Results in sliding autonomy for multi-robot spatial assembly. In: Proc. i-SAIRAS. pp. $448-455$.

Hoogendoorn, M., Gini, M., 2009. Preferences of agents in decentralized task allocation. AI Commun. 22 (3), 143-152.

Hsu, C.-I., Hung, S.-F., Li, H.-C., 2007. Vehicle routing problem with timewindows for perishable food delivery. Journal of Food Engineering 80 (2), 465-475. 
Hu, Q., Lim, A., 2014. An iterative three-component heuristic for the team orienteering problem with time windows. European Journal of Operational Research 232 (2), 276-286.

Hunsberger, L., Grosz, B., 2000. A combinatorial auction for collaborative planning. In: Proc. Int'l Conf on Multi-Agent Systems. pp. 151-158.

ILOG, I., 2006. ILOG CPLEX: High-performance software for mathematical programming and optimization.

URL http://www.ilog.com/products/cplex

Jackson, J., Faied, M., Kabamba, P., Girard, A., 2013. Distributed constrained minimum-time schedules in networks of arbitrary topology. IEEE Trans. on Robotics 29 (2), 554-563.

Jones, E. G., Dias, M. B., Stentz, A., 2011. Time-extended multi-robot coordination for domains with intra-path constraints. Autonomous Robots 30 (1), $41-56$.

Jozefowiez, N., Semet, F., Talbi, E.-G., 2008. Multi-objective vehicle routing problems. European Journal of Operational Research 189 (2), 293-309.

Jünger, M., Thienel, S., 2000. The ABACUS system for branch-and-cut-andprice algorithms in integer programming and combinatorial optimization. Software: Practice and Experience 30 (11), 1325-1352.

Junges, R., Bazzan, A. L. C., 2008. Evaluating the performance of DCOP algorithms in a real world, dynamic problem. In: Int'l Conf. on Autonomous Agents and Multi-Agent Systems. pp. 599-606.

Kang, K. H., Lee, Y. H., Lee, B. K., 2005. An exact algorithm for multi depot and multi period vehicle scheduling problem. In: Computational Science and Its Applications-ICCSA 2005. Springer, pp. 350-359.

Kitano, H., Satoshi, T., 2001. Robocup Rescue : A grand challenge for multiagent and intelligent systems. AI Magazine 22 (1), 39-52.

Kleiner, A., Farinelli, A., Ramchurn, S., Shi, B., Maffioletti, F., Reffato, R., 2013. RMASBench: Benchmarking dynamic multi-agent coordination in urban search and rescue. In: Int'l Conf. on Autonomous Agents and Multi-Agent Systems. pp. 1195-1196.

Koes, M., Nourbakhsh, I. R., Sycara, K. P., 2005. Heterogeneous multirobot coordination with spatial and temporal constraints. In: Proc. AAAI Conf. on Artificial Intelligence. pp. 1292-1297.

Kolen, A. W. J., Kan, A. H. G. R., Trienekens, H. W. J. M., 1987. Vehicle routing with time windows. Operations Research 35 (2), 266-273. 
Korsah, G. A., 2011. Exploring bounded optimal coordination for heterogeneous teams with cross-schedule dependencies. Ph.D. thesis, Robotics Institute, Carnegie Mellon University, Pittsburgh, PA.

Korsah, G. A., Kannan, B., Browning, B., Dias, M. B., 2012. xBots: An approach to generating and executing optimal multi-robot plans with constraints. In: Proc. IEEE Int'l Conf. on Robotics and Automation. pp. 115122 .

Korsah, G. A., Stentz, A., Dias, M. B., 2013. A comprehensive taxonomy for multi-robot task allocation. The International Journal of Robotics Research 32 (12), 1495-1512.

Labadie, N., Mansini, R., Melechovskỳ, J., Calvo, R. W., 2012. The team orienteering problem with time windows: An LP-based granular variable neighborhood search. European Journal of Operational Research 220 (1), 15-27.

Lagoudakis, M., Keskinocak, P., Kleywegt, A., Koenig, S., 2004. Auctions with performance guarantees for multi-robot task allocation. In: Proc. IEEE/RSJ Int. Conf. on Intelligent Robots and Systems. pp. 1957-1962.

Lagoudakis, M. G., Markakis, E., Kempe, D., Keskinocak, P., Kleywegt, A., Koenig, S., Tovey, C., Meyerson, A., Jain, S., 2005. Auction-based multirobot routing. In: Robotics: Science and Systems (RSS). pp. 343-350.

Landén, D., Heintz, F., Doherty, P., 2012. Complex task allocation in mixedinitiative delegation: A UAV case study. In: Desai, N., Liu, A., Winikoff, M. (Eds.), Principles and Practice of Multi-Agent Systems: 13th Int'l Conf., PRIMA 2010. Lecture Notes in Computer Science 7057. Springer, pp. 288303.

Laporte, G., Louveaux, F., Mercure, H., 1992. The vehicle routing problem with stochastic travel times. Transportation Science 26 (3), 161-170.

Lau, H. C., Sim, M., Teo, K. M., 2003. Vehicle routing problem with time windows and a limited number of vehicles. European Journal of Operational Research 148 (3), 559-569.

Lee, K., Choi, B.-C., Leung, J. Y. T., Pinedo, M. L., 2009. Approximation algorithms for multi-agent scheduling to minimize total weighted completion time. Inf. Process. Lett. 109 (16), 913-917.

Lerman, K., Jones, C., Galstyan, A., Matarić, M. J., 2006. Analysis of dynamic task allocation in multi-robot systems. The International Journal of Robotics Research 25 (3), 225-241.

Lombardi, M., Milano, M., 2012. Optimal methods for resource allocation and scheduling: a cross-disciplinary survey. Constraints 17 (1), 51-85. 
Luo, L., 2014. Distributed algorithm design for constrained multi-robot task assignment. Ph.D. thesis, Robotics Institute, Carnegie Mellon University, Pittsburgh, PA.

Luo, L., Chakraborty, N., Sycara, K., 2011. Multi-robot algorithm for tasks with set precedence constraints. In: Proc. IEEE Int'l Conf. on Robotics and Automation. pp. 2526-2533.

Luo, L., Chakraborty, N., Sycara, K., 2015. Distributed algorithms for multirobot task assignment with task deadline constraints. IEEE Trans. on Automation Science and Engineering 12 (3), 876-888.

Luo, Y., Schonfeld, P., 2007. A rejected-reinsertion heuristic for the static diala-ride problem. Transportation Research Part B: Methodological 41 (7), 736755 .

Macarthur, K. S., Stranders, R., Ramchurn, S. D., Jennings, N. R., 2011. A distributed anytime algorithm for dynamic task allocation in multi-agent systems. In: Proc. AAAI Conf. on Artificial Intelligence. pp. 701-706.

MacKenzie, D. C., 2003. Collaborative tasking of tightly constrained multi-robot missions. In: Multi-Robot Systems: From Swarms to Intelligent Automata, Volume II. Vol. 2. pp. 39-50.

Maheswaran, R. T., Tambe, M., Bowring, E., Pearce, J. P., Varakantham, P., 2004. Taking DCOP to the real world: Efficient complete solutions for distributed multi-event scheduling. In: Int'l Conf. on Autonomous Agents and Multi-Agent Systems. pp. 310-317.

Manne, A. S., 1960. On the job-shop scheduling problem. Operations Research 8 (2), 219-223.

McIntire, M., Nunes, E., Gini, M., 2016. Iterated multi-robot auctions for precedence-constrained task scheduling. In: Int'l Conf. on Autonomous Agents and Multi-Agent Systems. pp. 1078-1086.

Melvin, J., Keskinocak, P., Koenig, S., Tovey, C. A., Ozkaya, B. Y., 2007. Multirobot routing with rewards and disjoint time windows. In: Proc. IEEE/RSJ Int. Conf. on Intelligent Robots and Systems. pp. 2332-2337.

Mercker, T., Casbeer, D., Millet, P., Akella, M., 2010. An extension of consensus-based auction algorithms for decentralized, time-constrained task assignment. In: American Control Conf. pp. 6324-6329.

Miao, X., Luh, P., Kleimnman, D., Castanon, D., 1991. Distributed stochastic resource allocation in teams. IEEE Trans. on Systems, Man, and Cybernetics $21(1), 61-70$.

Mitiche, H., Bougaci, D., Gini, M., 2015a. Efficient heuristics for a timeextended multi-robot task allocation problem. In: International Conference on New Technologies of Information and Telecommunication. Mila, Algeria. 
Mitiche, H., Godoy, J., Gini, M., 2015b. On the tuning and evaluation of iterated local search. In: Proc. of Metaheuristics International Conference (MIC). Agadir, Morocco.

Monma, C. L., Apr. 1981. Sequencing with general precedence constraints. Discrete Applied Mathematics 3, 137-150.

Nanjanath, M., Gini, M., 2010. Repeated auctions for robust task execution by a robot team. Robotics and Autonomous Systems 58 (7), 900-909.

Nunes, E., Gini, M., 2015. Multi-robot auctions for allocation of tasks with temporal constraints. In: Proc. AAAI Conf. on Artificial Intelligence. pp. $2110-2116$.

Nunes, E., Nanjanath, M., Gini, M., 2012. Auctioning robotic tasks with overlapping time windows. In: Int'l Conf. on Autonomous Agents and Multi-Agent Systems. pp. 1211-1212.

Oddi, A., Rasconi, R., Cesta, A., Smith, S. F., 2011. Solving job shop scheduling with setup times through constraint-based iterative sampling: an experimental analysis. Annals of Mathematics and Artificial Intelligence 62 (3-4), 371-402.

Olawsky, D., Gini, M., 1990. Deferred planning and sensor use. In: Innovative Approaches to Planning, Scheduling and Control: Proc. 1990 DARPA Workshop. M. Kaufmann, San Mateo, Ca, pp. 166-174.

Parker, J., Gini, M., 2014. Tasks with cost growing over time and agent reallocation delays. In: Int'l Conf. on Autonomous Agents and Multi-Agent Systems. pp. 381-388.

Parker, J., Nunes, E., Godoy, J., Gini, M., 2016. Exploiting spatial locality and heterogeneity of agents for search and rescue teamwork. Journal of Field Robotics 33 (7), 877-900.

Parker, L. E., Tang, F., 2006. Building multi-robot coalitions through automated task solution synthesis. Proceedings of the IEEE 94 (7), 1289-1305.

Pavone, M., Bisnik, N., Frazzoli, E., Isler, V., 2009. A stochastic and dynamic vehicle routing problem with time windows and customer impatience. Mobile Networks and Applications 14 (3), 350-364.

Pavone, M., Frazzoli, E., Bullo, F., 2011. Adaptive and distributed algorithms for vehicle routing in a stochastic and dynamic environment. IEEE Trans. on Automatic Control 56 (6), 1259-1274.

Pecora, F., Cirillo, M., 2012. A constraint-based approach for multiple nonholonomic vehicle coordination in industrial scenarios. In: ICAPS 2012 Workshop on Combining Task and Motion Planning for Real-World Applications. pp. $45-52$. 
Planken, L. R., de Weerdt, M. M., van der Krogt, R. P., 2008. P3C: A new algorithm for the simple temporal problem. In: Proc. Int'l Conf. on Automated Planning and Scheduling. pp. 256-263.

Polacek, M., Hartl, R. F., Doerner, K., Reimann, M., 2004. A variable neighborhood search for the multi depot vehicle routing problem with time windows. Journal of Heuristics 10 (6), 613-627.

Ponda, S. S., 2012. Robust Distributed Planning Strategies for Autonomous Multi-Agent Teams. Ph.D. thesis, Massachusetts Institute of Technology, Department of Aeronautics and Astronautics.

Ponda, S. S., Johnson, L. B., How, J. P., 2012. Distributed chance-constrained task allocation for autonomous multi-agent teams. In: American Control Conf. pp. $4528-4533$.

Ponda, S. S., Redding, J., Choi, H.-L., How, J., Vavrina, M., Vian, J., 2010. Decentralized planning for complex missions with dynamic communication constraints. In: American Control Conf. pp. 3998-4003.

Pujol-Gonzalez, M., Cerquides, J., Farinelli, A., Meseguer, P., RodriguezAguilar, J. A., 2015. Efficient inter-team task allocation in RoboCup Rescue. In: Int'l Conf. on Autonomous Agents and Multi-Agent Systems. pp. 413-421.

Ramchurn, S., Farinelli, A., Macarthur, K., Polukarov, M., Jennings, N., 2010a. Decentralised coordination in RoboCup Rescue. The Computer Journal $53(9), 1-15$.

Ramchurn, S. D., Polukarov, M., Farinelli, A., Jennings, N., Trong, C., March 2010b. Coalition formation with spatial and temporal constraints. In: Int'l Conf. on Autonomous Agents and Multi-Agent Systems. pp. 1181-1188.

Ropke, S., Cordeau, J.-F., 2009. Branch and cut and price for the pickup and delivery problem with time windows. Transportation Science 43 (3), 267-286.

Ropke, S., Cordeau, J.-F., Laporte, G., 2007. Models and branch-and-cut algorithms for pickup and delivery problems with time windows. Networks 49 (4), $258-272$.

Rubinstein, Z., Smith, S., Barbulescu, L., 2012. Incremental management of oversubscribed vehicle schedules in dynamic dial-a-ride problems. In: Proc. AAAI Conf. on Artificial Intelligence. pp. 1809-1815.

Sandholm, T., Larson, K., Andersson, M., Shehory, O., Tohm, F., 1999. Coalition structure generation with worst case guarantees. Artificial Intelligence 111 (12), 209-238.

Sariel, S., Balch, T., 2006. A distributed multi-robot cooperation framework for real time task achievement. In: Gini, M., Voyles, R. (Eds.), Distributed Autonomous Robotic Systems. Springer Japan, pp. 187-196. 
Sariel-Talay, S., Balch, T., Erdogan, N., 2009. Multiple traveling robot problem: A solution based on dynamic task selection and robust execution. IEEE/ASME Trans. on Mechatronics 14 (2), 198-206.

Savelsbergh, M. W., Sol, M., 1995. The general pickup and delivery problem. Transportation science 29 (1), 17-29.

Savelsbergh, M. W. P., 1985. Local search in routing problems with time windows. Annals of Operations Research 4 (1), 285-305.

Scerri, P., Farinelli, A., Okamoto, S., Tambe, M., 2005. Allocating tasks in extreme teams. In: Int'l Conf. on Autonomous Agents and Multi-Agent Systems. pp. $727-734$.

Schneider, J., Apfelbaum, D., Bagnell, D., Simmons, R., 2005. Learning opportunity costs in multi-robot market based planners. In: Proc. IEEE Int'l Conf. on Robotics and Automation. pp. 1151-1156.

Segui-Gasco, P., Shin, H.-S., Tsourdos, A., Seguí, V., 2015. Decentralised submodular multi-robot task allocation. In: Proc. IEEE/RSJ Int. Conf. on Intelligent Robots and Systems. pp. 2829-2834.

Shah, J. A., Conrad, P. R., Williams, B. C., 2009. Fast distributed multi-agent plan execution with dynamic task assignment and scheduling. In: Proc. Int'l Conf. on Automated Planning and Scheduling. pp. 289-296.

Shehory, O., Kraus, S., 1998. Methods for task allocation via agent coalition formation. Artificial Intelligence 101 (1-2), 165-200.

Shen, Z., Ordónez, F., Dessouky, M. M., 2009. The stochastic vehicle routing problem for minimum unmet demand. In: Optimization and logistics challenges in the enterprise. Springer, pp. 349-371.

Smith, S. L., Bullo, F., 2007. Target assignment for robotic networks: Asymptotic performance under limited communication. In: American Control Conf. IEEE, pp. 1155-1160.

Solomon, M. M., 1986. On the worst case performance of some heuristics for the vehicle routing and scheduling problems with time window constraints. Networks 16, 161-174.

Solomon, M. M., Desrosiers, J., 1988. Survey paper - time window constrained routing and scheduling problems. Transportation Science 22 (1), 1-13.

Stergiou, K., Koubarakis, M., 2000. Backtracking algorithms for disjunctions of temporal constraints. Artificial Intelligence 120 (1), 81-117.

Su, X., Zhang, M., Bai, Q., 2016. Coordination for dynamic weighted task allocation in disaster environments with time, space and communication constraints. Journal of Parallel and Distributed Computing 97, 47-56. 
Taş, D., Dellaert, N., Van Woensel, T., De Kok, T., 2013. Vehicle routing problem with stochastic travel times including soft time windows and service costs. Comput. Oper. Res. 40 (1), 214-224.

Taillard, E., Badeau, P., Gendreau, M., Guertin, F., Potvin, J. Y., 1997. A tabu search heuristic for the vehicle routing problem with soft time windows. Transportation Science 31 (2), 170-186.

Tang, F., Parker, L. E., 2007. A complete methodology for generating multirobot task solutions using ASyMTRe-D and market-based task allocation. In: Proc. IEEE Int'l Conf. on Robotics and Automation. pp. 3351-3358.

Toth, P., Vigo, D. (Eds.), 2002. The vehicle routing problem. SIAM Monographs on Discrete Mathematics and Applications, Philadelphia, PA.

Tsamardinos, I., 2002. A probabilistic approach to robust execution of temporal plans with uncertainty. In: Methods and Applications of Artificial Intelligence: Proc. 2nd Hellenic Conference on AI (SETN '02). Lecture Notes in Computer Science. Springer-Verlag, pp. 97-108.

Usug, U. C., Sariel-Talay, S., 2011. Dynamic temporal planning for multirobot systems. In: Workshop on Automated Action Planning for Autonomous Mobile Robots at AAAI. pp. 64-69.

Vidal, T., 1999. Handling contingency in temporal constraint networks: from consistency to controllabilities. Journal of Experimental \& Theoretical Artificial Intelligence 11 (1), 23-45.

Vidal, T., Crainic, T., Gendreau, M., Prins, C., 2013. Heuristics for multiattribute vehicle routing problems: A survey and synthesis. European Journal of Operational Research 231 (1), 1-21.

Vidal, T., Crainic, T. G., Gendreau, M., Prins, C., 2014. Time-window relaxations in vehicle routing heuristics. Journal of Heuristics 21 (3), 329-358.

Vig, L., Adams, J. A., 2006. Multi-robot coalition formation. IEEE Trans. on Robotics 22 (4), 637-649.

Wu, F., Jennings, N., 2014. Regret-based multi-agent coordination with uncertain task rewards. In: Proc. AAAI Conf. on Artificial Intelligence. pp. 1492-1499.

Xu, L., Choueiry, B. Y., 2003. A new efficient algorithm for solving the simple temporal problem. In: Proc. of Int'l Symposium on Temporal Representation and Reasoning. pp. 212-222.

Xu, Y., Scerri, P., Yu, B., Okamoto, S., Lewis, M., Sycara, K., 2005. An integrated token-based algorithm for scalable coordination. In: Int'l Conf. on Autonomous Agents and Multi-Agent Systems. pp. 407-414. 
Zavlanos, M. M., Spesivtsev, L., Pappas, G. J., 2008. A distributed auction algorithm for the assignment problem. In: IEEE Proc. Decision and Control Conf. pp. $1212-1217$.

Zhang, Y., Parker, L. E., 2013a. IQ-ASyMTRe: Forming executable coalitions for tightly coupled multirobot tasks. IEEE Trans. on Robotics 29, 1-17.

Zhang, Y., Parker, L. E., 2013b. Multi-robot task scheduling. In: Proc. IEEE Int'l Conf. on Robotics and Automation. pp. 2992-2998.

Zheng, T., Woodside, M., 2003. Heuristic optimization of scheduling and allocation for distributed systems with soft deadlines. In: Computer Performance Evaluation. Modelling Techniques and Tools. Springer, pp. 169-181. 